\title{
Xanthine oxidase inhibitor ameliorates postischemic renal injury in mice by promoting resynthesis of adenine nucleotides
}

Kentaro Fujii, ${ }^{1}$ Akiko Kubo, ${ }^{2}$ Kazutoshi Miyashita, ${ }^{1}$ Masaaki Sato, ${ }^{1}$ Aika Hagiwara, ${ }^{1}$ Hiroyuki Inoue, ${ }^{1}$ Masaki Ryuzaki, ${ }^{1}$ Masanori Tamaki, ${ }^{1,3}$ Takako Hishiki, ${ }^{2,4}$ Noriyo Hayakawa, ${ }^{4}$ Yasuaki Kabe, ${ }^{2}$ Hiroshi Itoh, ${ }^{1}$ and Makoto Suematsu ${ }^{2}$

'Division of Endocrinology and Metabolism and Nephrology, Department of Internal Medicine and ${ }^{2}$ Department of Biochemistry, Keio University School of Medicine, Shinjuku-ku, Tokyo, Japan. ${ }^{3}$ Department of Nephrology, Graduate School of Medical Sciences, Tokushima University, Tokushima City, Tokushima, Japan. ${ }^{4}$ Clinical and Translational Research Center, Keio University School of Medicine, Shinjuku-ku, Tokyo, Japan.

Although oxidative stress plays central roles in postischemic renal injury, region-specific alterations in energy and redox metabolism caused by short-duration ischemia remain unknown. Imaging mass spectrometry enabled us to reveal spatial heterogeneity of energy and redox metabolites in the postischemic murine kidney. After 10-minute ischemia and 24-hour reperfusion (10mIR), in the cortex and outer stripes of the outer medulla, ATP substantially decreased, but not in the inner stripes of the outer medulla and inner medulla. $10 \mathrm{mIR}$ caused renal injury with elevation of fractional excretion of sodium, although histological damage by oxidative stress was limited. Ischemia-induced NADH elevation in the cortex indicated prolonged production of reactive oxygen species by xanthine oxidase (XOD). However, consumption of reduced glutathione after reperfusion suggested the amelioration of oxidative stress. An XOD inhibitor, febuxostat, which blocks the degradation pathway of adenine nucleotides, promoted ATP recovery and exerted renoprotective effects in the postischemic kidney. Because effects of febuxostat were canceled by silencing of the hypoxanthine phosphoribosyl transferase 1 gene in cultured tubular cells, mechanisms for the renoprotective effects appear to involve the purine salvage pathway, which uses hypoxanthine to resynthesize adenine nucleotides, including ATP. These findings suggest a novel therapeutic approach for acute ischemia/reperfusion renal injury with febuxostat through salvaging highenergy adenine nucleotides.

Authorship note: KF, AK, and KM contributed equally to this work.

Conflict of interest: The authors have declared that no conflict of interest exists.

Copyright: (c) 2019, American Society for Clinical Investigation.

Submitted: March 5, 2019

Accepted: October 10, 2019

Published: November 14, 2019

Reference information: /CI Insight. 2019:4(22):e124816.

https://doi.org/10.1172/jci.

insight.124816.

\section{Introduction}

Acute kidney injury (AKI), which is defined as an increase in creatinine levels by $0.3 \mathrm{mg} / \mathrm{dL}$ or more within 48 hours, or a decrease in urine volume to $0.5 \mathrm{~mL} / \mathrm{kg} / \mathrm{h}$ for 6 hours or longer (1), occurs in 3\%-20\% of hospitalized patients and is associated with high morbidity and mortality (2). Although primary causes of AKI include various conditions such as dehydration, sepsis, and nephrotoxicity, renal ischemia commonly promotes the progression of AKI (3). In a clinical setting, renal ischemia for less than 10 minutes can cause AKI. For example, renal ischemia because of cardiac arrest and resuscitation causes AKI in up to $30 \%$ of the patients $(4,5)$.

One of the principal mechanisms of renal ischemia/reperfusion (IR) injury is an excessive production of reactive oxygen species (ROS), which is generated through degradation of intracellular ATP and downstream adenine nucleotides with xanthine oxidase (XOD) (6). Treatments to suppress ROS during reperfusion have been attempted, but the effect of radical scavengers that can significantly reduce the tissue ROS level is not sufficient to ameliorate renal IR injury (7). The other potential mechanism of renal IR injury is ATP depletion in tubular cells. Shortage of ATP impairs the ATP-dependent tubular function and triggers multiple pathways of cellular injury that provoke necrosis and apoptosis as well as subsequent tissue inflammatory and fibrotic changes $(8,9)$. Shortage of adenine nucleotides other than ATP can also exacerbate renal IR injury. Inhibition of hydrolysis of adenosine monophosphate (AMP) protects the kidney from 
IR injury by maintaining the tissue AMP level $(10,11)$. Accelerated restoration of deprived ATP by supplementation of the purine base inhibits necrosis of tubular cells and promotes recovery of cell viability (12).

Based on these findings, both excessive ROS production and ATP depletion are assumed to play critical roles in the progression of renal injury during and after transient ischemia. Transient ischemia can cause the transition to chronic kidney disease (CKD) when the AKI episode occurs repeatedly $(13,14)$. Although elucidation of region-specific alterations in energy and redox metabolism under ischemia would have great impact on the development of a novel therapeutic approach for AKI, the precise course by which transient ischemia causes metabolic alterations in each functional region of the kidney has not been examined enough. In addition, previous studies examined IR injury in mice by using long-duration ischemia models, such as 45-minute IR. Therefore, the mechanism of AKI induced by short-duration ischemia, such as 10-minute IR, which is often seen in a clinical setting, remains unclear. Alterations of adenine nucleotides during and after transient ischemia have been evaluated using HPLC (15) or microdialysis technique (16) in the murine kidney. However, these conventional approaches require a complicated procedure for sample preparation that may degrade metabolites, and they are not appropriate for examining alterations of energy and redox metabolism after short-duration ischemia. Consequently, region-specific alterations of metabolites during and after renal ischemia remain unclear.

The alterations of adenine nucleotides under ischemia might be different among the regions of kidneys because spatial demands for substrates of energy metabolism are different. Proximal tubular cells lack glycolytic metabolism to avoid using reabsorbed glucose from the lumen, and therefore, the ATP production in the cortex mainly depends on mitochondrial respiration by using lactate and glutamine as substrates (17). On the other hand, the tubular cells in the medulla, such as those in the thick ascending limb (TALs) of loop of Henle and collecting ducts, contain glycolytic enzymes (18). Although the distribution of glycolytic enzymes in the kidney has been investigated, distributions of metabolites themselves have not been verified. Based on these facts, we examined metabolic alterations in each region of the murine kidney during and after transient ischemia by applying semiquantitative imaging mass spectrometry.

Matrix-assisted laser desorption/ionization-imaging mass spectrometry (MALDI-IMS) is a technology that has been used for visualizing in situ distributions of small metabolites (19). It has been used for examining tissue distributions of substances, which are difficult for immunostaining techniques to detect (20-23). We have successfully established a technique to semiquantitatively visualize distributions of lowmolecular weight metabolites by combining metabolome analyses and a snap-freezing procedure of murine organs to avoid degradation of metabolites (23). Using serial frozen sections, the technique allowed us to visualize distributions of ATP, ADP, AMP, adenosine, hypoxanthine, and xanthine in the liver and brain (20-25). Because kidneys have the capacity to excrete and reabsorb adenylates in a region-specific manner, examining heterogeneous responses of these metabolites among different regions would help us understand mechanisms for energy management during ischemia.

The present study aimed to clarify the region-specific mechanisms of renal IR injury caused by short-duration ischemia induced by renal artery clipping for 10 minutes. To clarify the spatial heterogeneity of energy and redox metabolism that accounts for the postischemic injury, MALDI-IMS combined with metabolomics was applied to the murine kidney. Xanthine dehydrogenase (XDH) and XOD, which are interconvertible proteins, play a crucial role in determining oxidative stress as well as the purine salvage pathway for ATP maintenance. Because inhibition of XOD has been reported to exert protective effects on ischemic myocardial or renal injury $(26,27)$, the mechanism of the action of febuxostat, a representative inhibitor of XOD, on amelioration of postischemic renal injury was examined.

\section{Results}

Transient renal ischemia for 10 minutes caused kidney dysfunction without apparent histological damage. To examine the impact of short-duration IR on renal function and oxidative damage, biochemical and histological examination were performed on the murine kidney with 10 minutes' ischemia followed by 24 hours' reperfusion (Figure 1A). An increase in $N$-acetyl- $\beta$-D-glucosaminidase (NAG) in urine and a decrease in creatinine clearance ( $\mathrm{CCr}$ ) were observed in the IR mice with renal ischemia for 10 minutes. Transient ischemia for a minute did not cause such renal damage (Figure 1B). The brush border of the proximal tubular cells was well maintained, and infiltration of inflammatory cells was not evident after 10 minutes' ischemia and 24 hours' reperfusion. The increase of oxidative stress marker 8-hydroxydeoxyguanosine (8-OHdG) was not significant as judged immunohistochemically (Figure 1, C-E). In contrast, an increase in the urine 
A

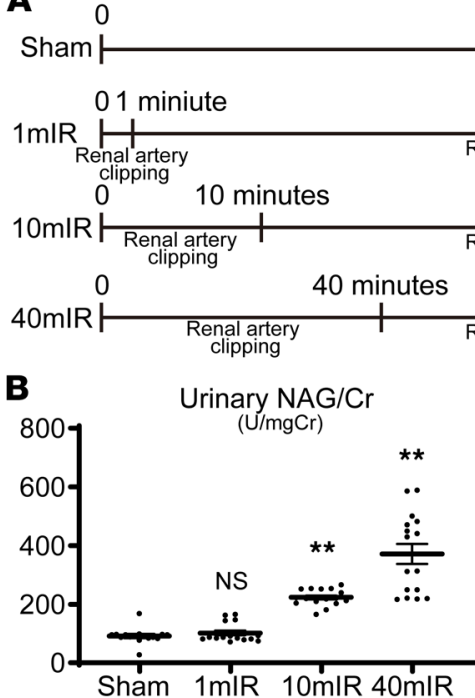

24 hours
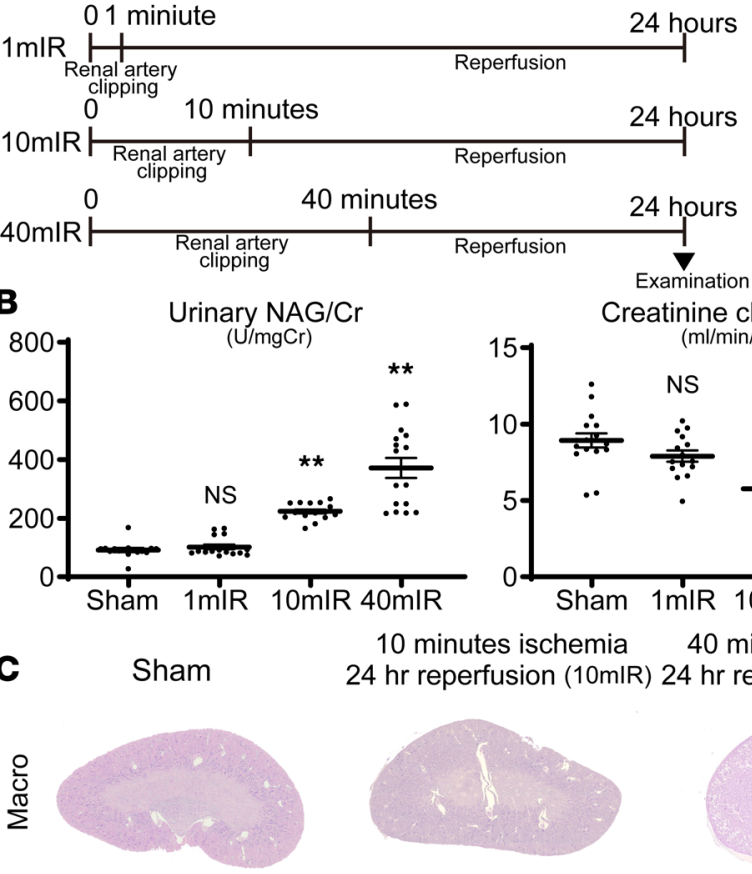

10 minutes ischemia

Creatinine clearance

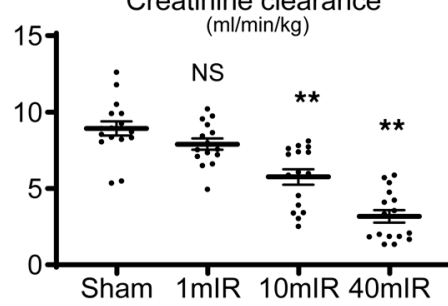
$24 \mathrm{hr}$ reperfusion (10mIR) $24 \mathrm{hr}$ reperfusion $(40 \mathrm{mIR})$
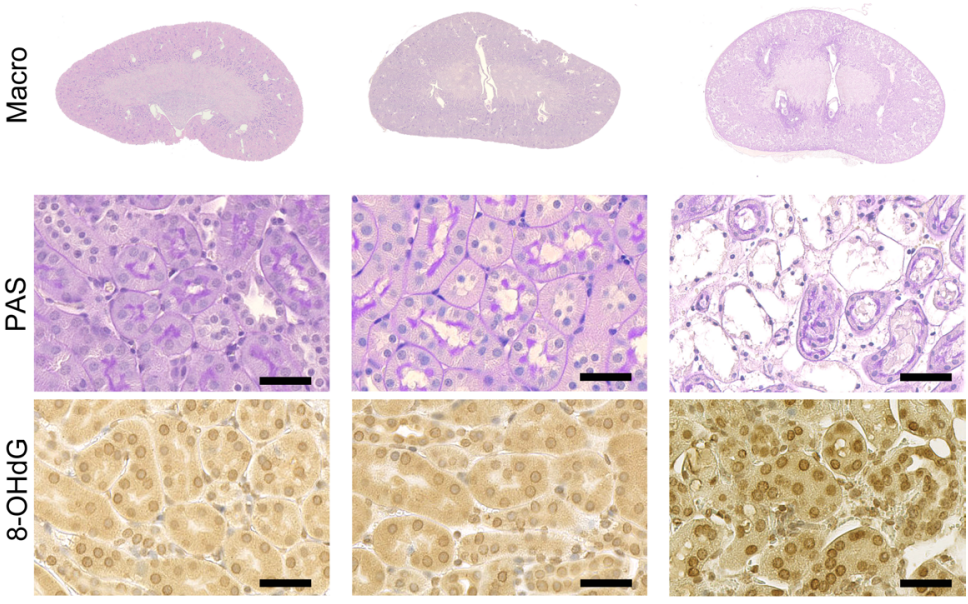

D

Tissue injury score
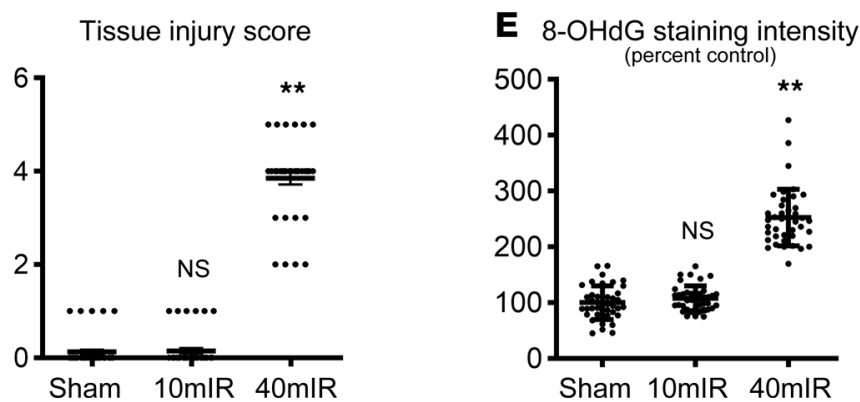

Figure 1. Transient renal ischemia for $\mathbf{1 0}$ minutes caused kidney dysfunction without apparent histological damage. (A) Scheme of the present study. (B) The ratio of urinary $N$-acetyl- $\beta$-D-glucosaminidase (NAG) to urinary creatinine (urinary NAG/Cr) and creatinine clearance. NS, not significant $(P>0.05) .{ }^{* *} P<0.01$ vs. sham. $n=16$ animals for each group. Differences in mean values among 4 groups (sham, $1 \mathrm{mIR}, 10 \mathrm{mIR}, 40 \mathrm{mIR}$ ) were statistically analyzed by 1-way ANOVA and Tukey's post hoc test. (C) Tissue injury by periodic acid-Schiff stain and oxidative stress by 8-OHdG stain were evaluated histologically. Quantification of the sections was shown as follows. (D) Tubulointerstitial injury score in the cortex. NS, not significant $(P>0.05) .{ }^{* *} P<0.01$ vs. sham. $n=4$ animals, 10 fields/animal for each group. (E) Oxidative damage in the cortex was estimated by 8-OHdG intensity of nuclei. NS, not significant $(P>0.05) .{ }^{*} P<0.01$ vs. sham. $n=4$ animals, 10 nuclei/animal for each group. Differences of means between the 2 groups were statistically analyzed by Student's 2-tailed $t$ test. $1 \mathrm{mIR}, 1$-minute ischemia and 24-hour reperfusion. $10 \mathrm{mIR}$, 10 -minute ischemia and 24-hour reperfusion. $40 \mathrm{mIR}, 40$-minute ischemia and 24-hour reperfusion. 8-OHdG, 8-hydroxydeoxyguanosine. Scale bar: $20 \mu \mathrm{m}$.

NAG level and a decrease in CCr were more apparent in the IR mice with renal ischemia for 40 minutes (Figure 1B), as shown in a previous report (28). Loss of the apical brush border in the proximal tubular cells and an increase in the density of $8-\mathrm{OHdG}$ staining were also apparent in the IR mice with 40 minutes' ischemia (Figure 1, C-E). These data indicate that short-duration renal ischemia for 10 minutes caused AKI without significant histological change or apparent oxidative damage, while the latter were evident in the IR mice with renal ischemia for 40 minutes.

Region-specific distribution of low-molecular weight metabolites in the normal kidney was successfully visualized by using MALDI-IMS. To evaluate the distribution of various low-molecular weight metabolites in the kidney, MALDI-IMS was applied in the sections of normal murine kidneys that were snap-frozen within a second after the dissection. Principal metabolites in the glycolytic pathway, tricarboxylic acid (TCA) cycle, 
Table 1. The contents of adenine nucleotides in the whole kidney quantified by metabolome analyses

\begin{tabular}{lccccc}
\hline & ATP & ADP & AMP & Total & EC value \\
Intact & $2801 \pm 158$ & $598 \pm 38$ & $251 \pm 29$ & $3649 \pm 186$ & $0.80 \pm 0.01$ \\
1-minute ischemia & $1583 \pm 46$ & $874 \pm 48$ & $999 \pm 94$ & $3455 \pm 168$ & $0.60 \pm 0.01$ \\
10-minute ischemia & $482 \pm 53$ & $444 \pm 31$ & $1455 \pm 130$ & $2381 \pm 197$ & $0.51 \pm 0.01$
\end{tabular}

$n=5$ animals for each group. The values are shown as mean \pm SEM in micromoles. Total, total adenylates (ATP + ADP + AMP); EC value, energy charge value $(A T P+[0.5 \times A D P] /[A T P+A D P+A M P])$.

amino acids, and adenine nucleotides were successfully visualized by using MALDI-IMS (Supplemental Table 1 and Supplemental Figures 1 and 2; supplemental material available online with this article; https:// doi.org/10.1172/jci.insight.124816DS1). The areas of the renal cortex, outer stripes of outer medulla (OSOM), inner stripes of outer medulla (ISOM), and inner medulla were determined by the histological characteristics (Supplemental Figure 3).

Metabolites of the TCA cycle, such as malate, showed cortex-dominant and outer medulla-dominant distribution, where mitochondria exist richly and the energy metabolism is reported to be active. Intermediate metabolites in the glycolytic pathway were predominantly observed in the ISOM, where glycolytic enzymes are rich (18). Creatinine, which is concentrated in the medulla, especially localized in the inner medulla. Osmotic regulator glycerophosphocholine, which was reported to localize in the medulla (29), was found in the inner medulla and ISOM; 2,3-bisphosphoglyceric acid, which is concentrated in erythrocytes, was localized in the vessels (Supplemental Figure 2). In these ways, the regional distribution of metabolites in the kidney was successfully visualized by using MALDI-IMS.

The region-specific content of adenine nucleotides and the breakdown products were semiquantitatively estimated by combining MALDI-IMS and metabolome analyses. To determine the content of adenine nucleotides (ATP, ADP, AMP), the breakdown products (inosine monophosphate [IMP], adenosine, inosine, hypoxanthine, xanthine, uric acid), and phosphoribosyl pyrophosphate (PRPP) in the whole kidney, we conducted capillary electrophoresis-MS-based (CE-MS-based) metabolome analysis under normal and ischemic conditions induced by renal artery clipping for 1 or 10 minutes (Tables $1-3$ ). The signal intensity of adenine nucleotides and the breakdown products in the murine kidney was examined by MALDI-IMS in the normal and ischemic sections. The semiquantitative content of each molecule in each region, that is, the cortex, OSOM, ISOM, and inner medulla, was determined from the signal intensity of MALDI-IMS, relative volume of each region, and content of each molecule in the whole kidney quantified by metabolome analyses (see Methods for more detail) (23). In these ways, the apparent content of these molecules in each region and the alteration during ischemia were successfully estimated and visualized in the cortex, OSOM, ISOM, and inner medulla (Figure 2 and Supplemental Tables 2-4). Such a semiquantitative technique is necessary for intersample comparisons of the content of molecules, because the signal intensity of MALDI-IMS itself does not accurately reflect the content.

In the normal kidney sections, high-energy adenine nucleotides (ATP and ADP) were significantly rich $(P<0.05$ vs inner medulla) in both the cortex and the outer medulla (OSOM and ISOM). The ATP-rich regions were in good agreement with the area where ATP production has been reported to be active (17). Adenosine, a breakdown product of ATP, has been believed to accumulate in the area where degradation or consumption of ATP is increased and the energy charge value, which is an index of tissue energy level, defined as $(\mathrm{ATP}+[0.5 \times \mathrm{ADP}]) /(\mathrm{ATP}+\mathrm{ADP}+\mathrm{AMP})$, is low

Table 2. The breakdown products in the whole kidney quantified by metabolome analyses

\begin{tabular}{|c|c|c|c|c|c|}
\hline & Adenosine & Inosine & Hypoxanthine & Xanthine & Uric acid \\
\hline Intact & $37.5 \pm 7.3$ & $14.0 \pm 3.4$ & $5.3 \pm 1.3$ & $2.8 \pm 1.7$ & $20.5 \pm 5.5$ \\
\hline 1-minute ischemia & $66.0 \pm 5.5$ & $54 \pm 3.7$ & $47.9 \pm 2.0$ & $27.4 \pm 2.5$ & $30.4 \pm 3.3$ \\
\hline 10-minute ischemia & $87.8 \pm 3.6$ & $252 \pm 17.5$ & $250.5 \pm 11.8$ & $117.5 \pm 7.5$ & $68.4 \pm 12.2$ \\
\hline
\end{tabular}

$n=5$ animals for each group. The values are shown as mean \pm SEM in micromoles. 
Table 3. Purine biosynthesis intermediates in the whole kidney quantified by metabolome analyses

\begin{tabular}{lcc}
\hline & PRPP & IMP \\
Intact & $5.3 \pm 0.8$ & $69.3 \pm 10.0$ \\
1-minute ischemia & $4.6 \pm 0.9$ & $289.7 \pm 14.9$ \\
10-minute ischemia & $2.8 \pm 0.3$ & $673.0 \pm 36.0$
\end{tabular}

$n=5$ animals for each group. The values are shown as mean \pm SEM in micromoles. PRPP, phosphoribosyl pyrophosphate; IMP, inosine monophosphate.

Meanwhile, the present study revealed that adenosine specifically accumulated in the OSOM. Adenosine specifically accumulated in the OSOM of the normal kidney, which was clarified for the first time by the techniques using MALDI-IMS. Adenosine is a breakdown product of ATP that increases in the ischemic region, in which ATP is dephosphorylated to ADP, AMP, and adenosine (30). Because the region that contains the OSOM is known to be a relatively ischemic area $(31,32)$ because of the great demand of ATP for active transportation by the S3 segment of the proximal tubule, it is possible that the adenosine in the OSOM was accumulated by the degradation of ATP. However, the other degradative products of ATP, namely, ADP and AMP, were not increased, and the energy charge value was high in the OSOM. The other breakdown products, such as inosine and hypoxanthine, could hardly be observed because their concentrations seemed low (Figure 2 and Supplemental Tables 2-4).

ATP and total adenylates in the cortex and OSOM decreased by transient ischemia for 10 minutes. In the sections exposed to ischemia by renal artery clipping, ATP in the cortex and the outer medulla decreased within a minute after the clipping procedure, and consequently, the breakdown products, including adenosine, inosine, and hypoxanthine, increased in every region of the kidney. In particular, the content of ATP decreased by $45 \%$ within a minute of ischemia, and the decrease reached $84 \%$ during 10 minutes' ischemia. In the inner medulla, in contrast, ATP did not decrease within a minute, and significant decline $(P<0.01)$ first became evident at 10 minutes after the clipping procedure. Conversely, AMP increased in every region of the kidney during the ischemia. Energy charge value decreased in the whole kidney within a minute. Total adenylates, defined as ATP + ADP + AMP, decreased significantly $(P<0.01)$ in the cortex and OSOM within a minute, and the depletion became prominent at 10 minutes after promoting ischemia. Nevertheless, total adenylates in the ISOM and inner medulla were not altered by 10 minutes' ischemia. The physiological accumulation of adenosine in the OSOM disappeared after the clipping procedure, which was associated with the increase of adenosine in regions of the kidney except for the OSOM. The breakdown products inosine and hypoxanthine increased in every region of the kidney within a minute and in parallel to AMP, and the degrading changes of adenylates progressed during 10 minutes' ischemia. Although xanthine and uric acid were difficult to detect with MALDI-IMS, CE-MS-based metabolome analysis revealed the increase in the ischemic kidney (Table 2). In these ways, region-specific alterations of adenine nucleotides and their degrading products during transient ischemia for 1 to 10 minutes was successfully visualized in the murine kidney (Figure 2 and Supplemental Tables 2-4).

To examine the restoration of ATP by 24 hours' reperfusion after transient ischemia, the content of adenine nucleotides and the breakdown products in the kidney were estimated by MALDI-IMS and CE-MS in the sham-operated mice and the 10 minutes' ischemia and 24 hours' reperfusion (IR) sections (Tables 4-6, Figure 3, and Supplemental Tables 5-7). In the kidneys of sham-operated mice, ATP in the ISOM tended to decrease, and adenosine in the ISOM tended to increase when compared with the normal mice; however, the content of ATP and the breakdown products were equivalent in the cortex. In the reperfusion sections, restoration of ATP in the renal cortex and OSOM was not complete, and ATP showed a $24 \%$ decrease when compared with sham sections. In contrast, the restoration of ATP in the ISOM and inner medulla was sufficient. Of note, total adenylates in the cortex and OSOM also decreased even after 24 hours' reperfusion, despite total adenylates being maintained in the ISOM and inner medulla. That is, total adenylates and ATP in the cortex and OSOM after 24 hours' reperfusion demonstrated prolonged loss. The breakdown products of ATP, which were increased in the whole kidney by 10 minutes' ischemia, almost recovered to the original content after 24 hours' reperfusion. 


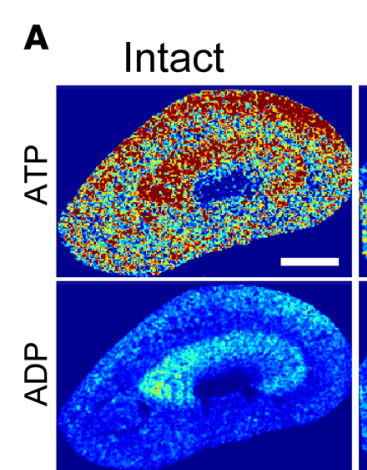

\section{1 minute ischemia \\ 10 minutes ischemia}
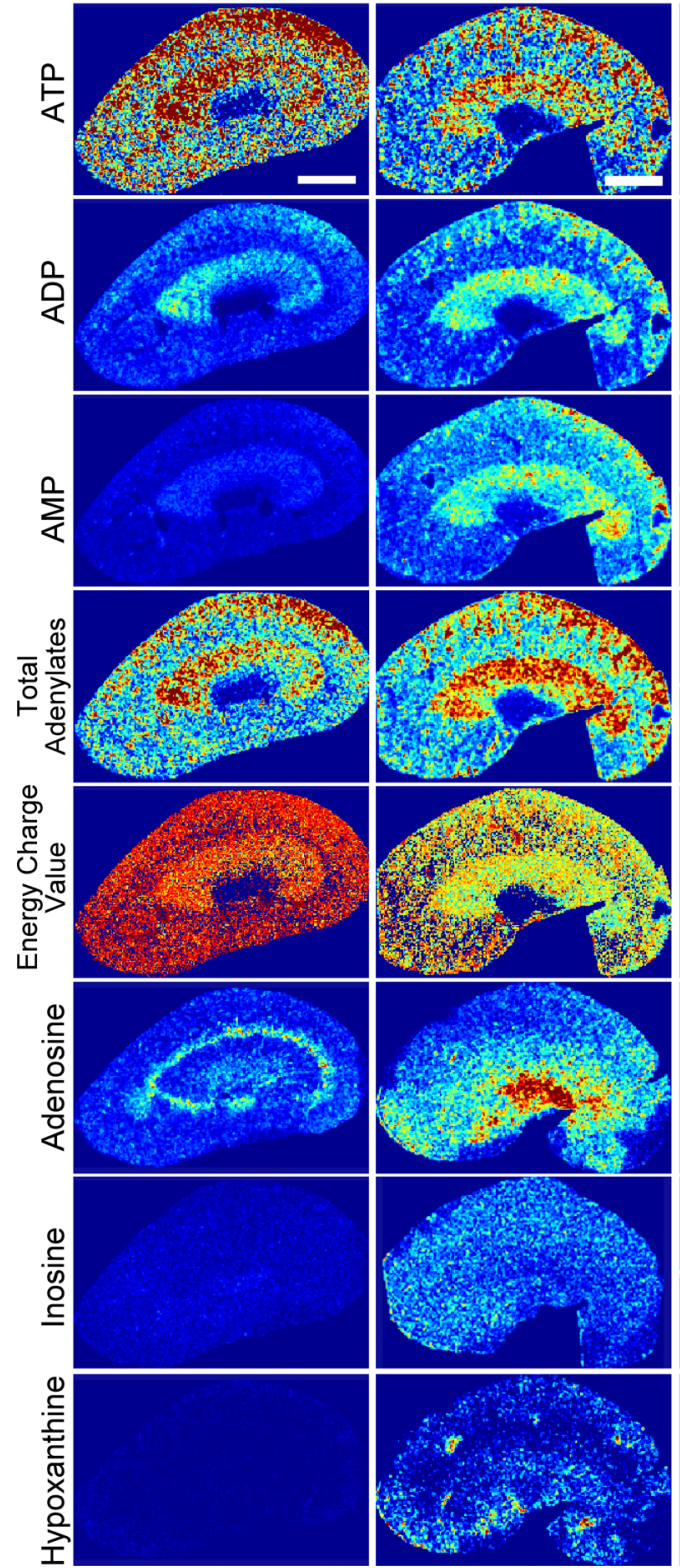
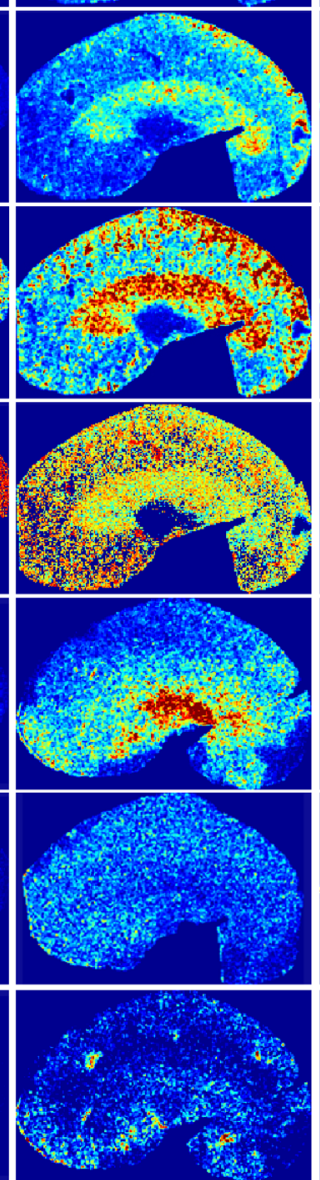
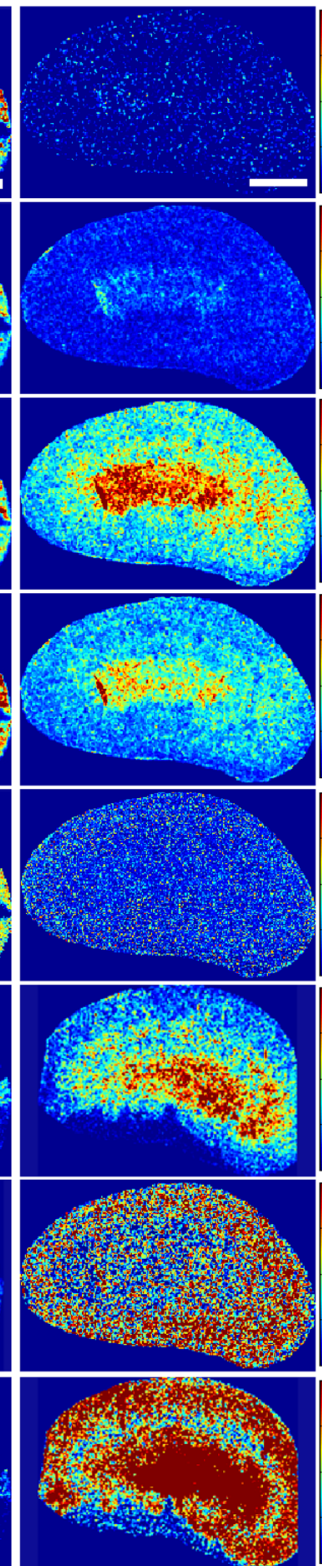

B

Intact $\square 1$ minute ischemia 10 minutes ischemia
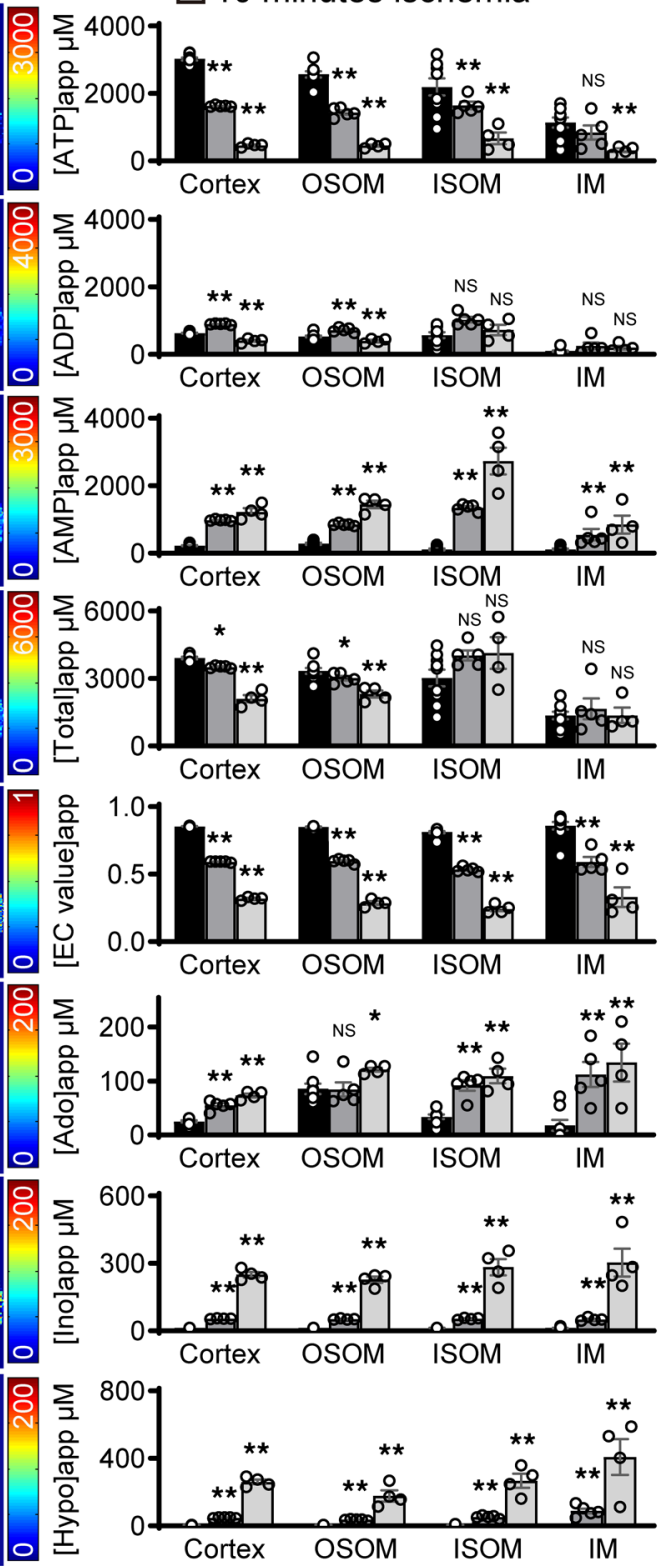

Figure 2. The region-specific contents of adenine nucleotides and their breakdown products assessed by semiquantitative MALDI-IMS. (A) Semiquantitative mass spectrometric images of snap-frozen normal murine kidney (left) and that in 1-minute (middle) and 10-minute ischemia (right) are reconstructed at a 35- $\mu \mathrm{m}$ resolution. (B) The apparent content of metabolites (mean \pm SEM) among the data points of the renal cortex, OSOM, ISOM, and IM. ${ }^{*} P<0.05,{ }^{* *} P<0.01$ vs. control. NS, not significant $(P>0.05)$. Scale bars: $1 \mathrm{~mm} . n=5$ animals in each group for quantitative analyses, and $n=9$ (intact). $n=5$ (1-minute ischemia). $n=4$ (10-minute ischemia) for imaging MS analyses. Differences in mean values among 3 groups (intact, 1-minute ischemia, 10-minute ischemia) were statistically analyzed by 1-way ANOVA and Tukey's post hoc test. OSOM, outer stripes of outer medulla; ISOM, inner stripes of outer medulla; IM, inner medulla; Ado, adenosine; Ino, inosine; Hypo, hypoxanthine.

Febuxostat promoted ATP recovery in the cortex and OSOM after short-duration ischemia. Although oxidative damage was not apparent in the murine kidney after transient ischemia for 10 minutes (Figure 1), a prolonged loss of ATP was significant after short-duration ischemia (Figure 3). Therefore, we hypothesized that ATP depletion contributed to the progression of the postischemic AKI. An XOD inhibitor, febuxostat, blocks the degradation pathway of ATP and increases hypoxanthine, which acts as a substrate for the resynthesis of adenine nucleotides. The effect of febuxostat can increase tissue ATP levels and ameliorate postischemic injury (33-35).

To examine the effects of febuxostat on metabolic alterations in the kidney after short-duration ischemia, we used semiquantitative MALDI-IMS to visualize adenine nucleotides and the breakdown products 
Table 4. The contents of adenine nucleotides after the 10-minute ischemia and 24-hour reperfusion in the whole kidney quantified by metabolome analyses

\begin{tabular}{lccccc}
\hline & ATP & ADP & AMP & Total & EC value \\
Sham & $2768 \pm 144$ & $702 \pm 56$ & $390 \pm 74$ & $3860 \pm 73$ & $0.81 \pm 0.03$ \\
IR & $2119 \pm 104$ & $617 \pm 45$ & $375 \pm 69$ & $3110 \pm 24$ & $0.78 \pm 0.03$ \\
IRF & $3194 \pm 125$ & $684 \pm 58$ & $293 \pm 50$ & $4170 \pm 211$ & $0.85 \pm 0.01$
\end{tabular}

$n=5$ animals for each group. The values are shown as mean \pm SEM in micromoles. Total, total adenylates (ATP + ADP + AMP); EC value, energy charge value (ATP + [0.5 × ADP]/[ATP + ADP + AMP]); IR, ischemia reperfusion; IRF, ischemia reperfusion with febuxostat treatment.

in each region of the murine kidney. Mice were given febuxostat in drinking water after transient renal artery clipping. The content of ATP in the cortex and OSOM of the kidney after 10 minutes' ischemia and 24 hours' reperfusion with the febuxostat treatment (IRF) was significantly higher than that of the kidney after the ischemia/reperfusion without febuxostat (IR) (Figure 3 and Supplemental Tables 5-7). Total adenylates in the cortex and OSOM that were depleted even after the 24-hour reperfusion were notably recovered by the febuxostat treatment. In the IRF mice sections, the content of hypoxanthine in every region of the kidney increased significantly $(P<0.01)$ compared with that in the IR mice, which showed the effect of febuxostat to inhibit degradation of hypoxanthine. In these ways, febuxostat boosted the recovery of total adenylates and ATP in the cortex and OSOM that was depleted by transient ischemia.

Redox mediators indicated ROS production in the postischemic kidney. To examine redox metabolism in the postischemic kidney by MALDI-IMS, we evaluated reduced glutathione (GSH), oxidized glutathione, and NADH levels, which are deeply associated with ROS production after ischemia (Figure 4 and Supplemental Table 8). Because the molecules that consist of ROS itself are too small to detect with MALDI-IMS, we inspected these redox-related molecules. $\mathrm{XDH}$ and XOD are same protein converting each other and degrading purines. NADH inhibits non-ROS-producing form $\mathrm{XDH}$ and converts it to ROS-producing form XOD. An ischemia-induced increase in NADH converts XDH to XOD and provokes production of hydrogen peroxide $\left(\mathrm{H}_{2} \mathrm{O}_{2}\right)$ through degradation of hypoxanthine and xanthine by XOD. That is, increases in NADH and hypoxanthine during ischemia account for the principal mechanism of ROS production after ischemia/reperfusion by XOD in the kidney (36). GSH is an antioxidant whose level increases by oxidative stimuli, and it detoxifies ROS with the sulfhydryl group (37). Ischemia/reperfusion-induced $\mathrm{H}_{2} \mathrm{O}_{2}$ is detoxified by GSH, preventing it from causing oxidative injury. Thus, NADH and GSH are key molecules that determine postischemic oxidative damage.

MALDI-IMS demonstrated that the GSH level increased more than 2 times after 10 minutes' ischemia. It dramatically decreased in each region of the kidney during 24 hours' reperfusion. These findings imply ROS production in the postischemic kidney and detoxification by GSH. NADH showed a 5 -fold increase in all functional regions of the kidney subjected to 10 minutes' ischemia. The increase in NADH in the cortex and OSOM persisted during 24 hours of reperfusion, whereas it significantly decreased $(P<$ 0.01 vs 10-minute ischemia) in the ISOM and inner medulla. The sustained elevation of NADH during reperfusion in the cortex and OSOM, but not in the ISOM and inner medulla, suggested cortex-specific ROS production throughout the period. In these ways, inspections of the redox mediators by MALDI-IMS

Table 5. The breakdown product after the 10-minute ischemia and 24-hour reperfusion in the whole kidney quantified by metabolome analyses

\begin{tabular}{lccccc}
\hline & Adenosine & Inosine & Hypoxanthine & Xanthine & Uric acid \\
Sham & $45.5 \pm 6.0$ & $25.4 \pm 3.3$ & $12.2 \pm 2.1$ & $9.4 \pm 1.2$ & $31.3 \pm 3.5$ \\
IR & $48.1 \pm 3.0$ & $19.3 \pm 3.7$ & $11.7 \pm 3.3$ & $7.6 \pm 1.7$ & $16.8 \pm 2.0$ \\
IRF & $24.8 \pm 4.6$ & $18.3 \pm 3.9$ & $38.6 \pm 3.2$ & $123.4 \pm 45.9$ & $22.7 \pm 1.6$
\end{tabular}

$n=5$ animals for each group. The values are shown as mean \pm SEM in micromoles. IR, ischemia reperfusion; IRF, ischemia reperfusion with febuxostat treatment. 
Table 6. Purine biosynthesis intermediates after the 10-minute ischemia and 24-hour reperfusion in the whole kidney quantified by metabolome analyses

\begin{tabular}{lcc}
\hline & PRPP & IMP \\
Sham & $6.9 \pm 0.4$ & $130.5 \pm 19.0$ \\
IR & $6.4 \pm 0.3$ & $107.8 \pm 25.0$ \\
IRF & $7.3 \pm 0.3$ & $109.4 \pm 23.5$
\end{tabular}

$n=5$ animals for each group. The values are shown as mean \pm SEM in micromoles. IR, ischemia reperfusion; IRF, ischemia reperfusion with febuxostat treatment; PRPP, phosphoribosyl pyrophosphate; IMP, inosine monophosphate.

suggested ROS production even after short-duration ischemia. However, evident oxidative damage was not observed, as judged by 8-OHdG staining (Figure 1, C and E). Febuxostat treatment significantly increased the GSH level after reperfusion, suggesting that the consumption of GSH during reperfusion was reduced by febuxostat. The findings implied that, in the cortex and OSOM, febuxostat treatment attenuates regional oxidative stress through its action on xanthine oxidase as an ROS generator after transient ischemia.

Febuxostat exerted protective effects against postischemic renal injury. To examine the effect of febuxostat on renal function after transient ischemia, biochemical examination was performed in the IR and IRF mice (Figure 5A). The oxidative stress marker, urinary $8-\mathrm{OHdG}$, did not increase in the IR mice, and the level was equivalent in the IRF mice (Figure $5 \mathrm{~B}$ ). Nevertheless, the ischemia/reperfusion procedure increased the cortical tubular injury marker, urinary NAG, and febuxostat suppressed the increase in NAG. Consequently, a reduction in $\mathrm{CCr}$ with a prominent increase in fractional excretion of sodium (FENa), which indicates an impairment in the ATP-dependent tubular function, in the IR mice were suppressed by the febuxostat treatment. Because the alteration of the 8 -OHdG level by short-duration ischemia was limited, the renoprotective effect of febuxostat seems to be exerted through a different mechanism from the antioxidative action. Meanwhile, the increase in FENa by short-duration ischemia was recovered by febuxostat, suggesting that the renoprotective effects of febuxostat are dependent on the promotion of ATP recovery.

Renoprotective roles of febuxostat against acute ischemia were examined by its administration at 20 minutes before or 10 minutes after transient ischemia (Figure 6A). The deteriorations of $\mathrm{CCr}$, urinary NAG, and FENa by the ischemia were significantly ameliorated by the treatment with febuxostat at both timings. The effect was more apparent when febuxostat was administrated after the ischemia (Figure 6B). These results suggested that febuxostat exerted renoprotective effects by boosting ATP recovery after ischemia rather than by suppressing ATP loss during ischemia.

Febuxostat promoted ATP recovery after oxygen and glucose deprivation in cultured tubular cells. The present experiments using the murine kidney indicated that febuxostat relieved renal injury after short-duration ischemia by boosting ATP recovery during reperfusion. A purine salvage pathway is a resynthesis pathway for adenine nucleotides, including ATP, by the action of hypoxanthine phosphoribosyl transferase 1 (HPRT1), which synthesizes IMP from hypoxanthine and PRPP (Supplemental Figure 4A). The finding that hypoxanthine did not increase as much as xanthine by febuxostat treatment after short-duration ischemia (Table 5) suggested that hypoxanthine had been used for the resynthesis of adenine nucleotides via the salvage pathway. Therefore, we hypothesized that suppression of XOD by febuxostat increases the hypoxanthine pool and thus promotes resynthesis of adenine nucleotides, thereby boosting ATP recovery after transient ischemia.

To investigate the mechanism by which febuxostat exerts renoprotective effects, we performed in vitro experiments using HK-2 tubular epithelial cells subjected to oxygen and glucose deprivation (OGD). HK-2 cells were exposed to hypoxia $\left(5 \% \mathrm{CO}_{2}, 1 \% \mathrm{O}_{2}\right)$ for 12 hours followed by 16 hours of reoxygenation $\left(5 \% \mathrm{CO}_{2}, 21 \% \mathrm{O}_{2}\right)$. During hypoxia, the culture medium was replaced with DMEM containing no glucose (OGD). The OGD and reoxygenation procedure is used as an in vitro model of IR injury in previous reports (12). ATP and $\mathrm{H}_{2} \mathrm{O}_{2}$ levels during and after OGD and the expression of renal injury markers was examined (Supplemental Figure 4B). OGD for 12 hours and reoxygenation for 16 hours decreased intracellular ATP by $20 \%$ and thus mimicked in vivo condition of the IR injury (Supplemental Figure 4C). Febuxostat treatment during reoxygenation efficiently recovered intracellular ATP and suppressed the increase in $\mathrm{H}_{2} \mathrm{O}_{2}$ after OGD and reoxygenation (Supplemental Figure 4, C and D). The expression of apoptosis-related genes CASP3 and KIM- 1 in the HK-2 cells was increased by OGD (Supplemental Figure 4E) and suppressed by febuxostat treatment (Supplemental Figure 4F). 


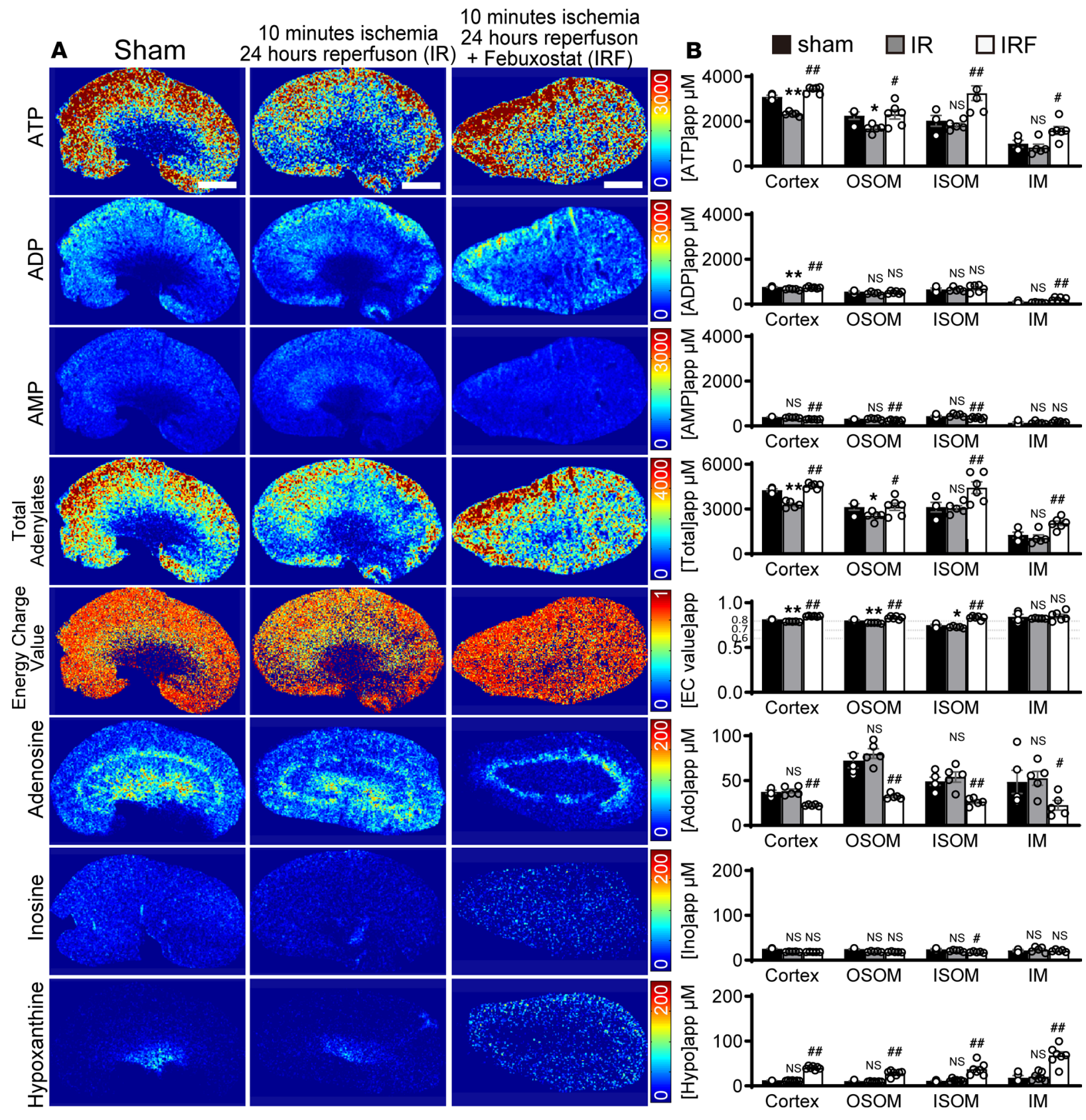

Figure 3. Febuxostat promoted ATP recovery in the cortex after short-duration ischemia. (A) Semiquantitative mass spectrometric images of snap-frozen sham-operated murine kidney (left), IR (middle), and IRF (right) are reconstructed at a 35- $\mu \mathrm{m}$ resolution. (B) The apparent contents of metabolites (mean \pm SEM) among the data points of the renal cortex, OSOM, ISOM, and IM. ${ }^{*} P<0.05$, ${ }^{*} P<0.01$ sham vs. IR. ${ }^{*} P<0.05$, $\# \# P<0.01$ IR vs. IRF. NS, not significant $(P>0.05)$. Scale bars: $1 \mathrm{~mm} . n=5$ animals in each group for quantitative analyses. $n=4$ (sham), $n=5$ (IR, IRF) for imaging MS analyses. Differences in mean values among 3 groups (sham, IR, IRF) were statistically analyzed by 1-way ANOVA and Tukey's post hoc test.

To elucidate the involvement of the purine salvage pathway in the promotion of ATP recovery and renoprotection by febuxostat treatment in $\mathrm{HK}-2$ cells, $100 \mu \mathrm{M}$ of hypoxanthine $-{ }^{13} \mathrm{C}_{2},{ }^{15} \mathrm{~N}$ was added during reoxygenation (Figure 7, A and B). ATP- ${ }^{13} \mathrm{C}_{2},{ }^{15} \mathrm{~N}$, which was generated via the salvage pathway, accounted for approximately $40 \%$ of total ATP. The total ATP amount was significantly increased by febuxostat treatment (Figure 7, C and D). That is, the salvage pathway works in HK-2 cells, and febuxostat promoted the resynthesis of adenine nucleotides, including ATP.

Febuxostat promoted resynthesis of adenine nucleotides via the HPRT1-dependent purine salvage pathway and suppressed the renal injury marker genes. To examine the significance of the purine salvage pathway in the resynthesis of adenine nucleotides and renoprotection by febuxostat treatment, genetic silencing of 

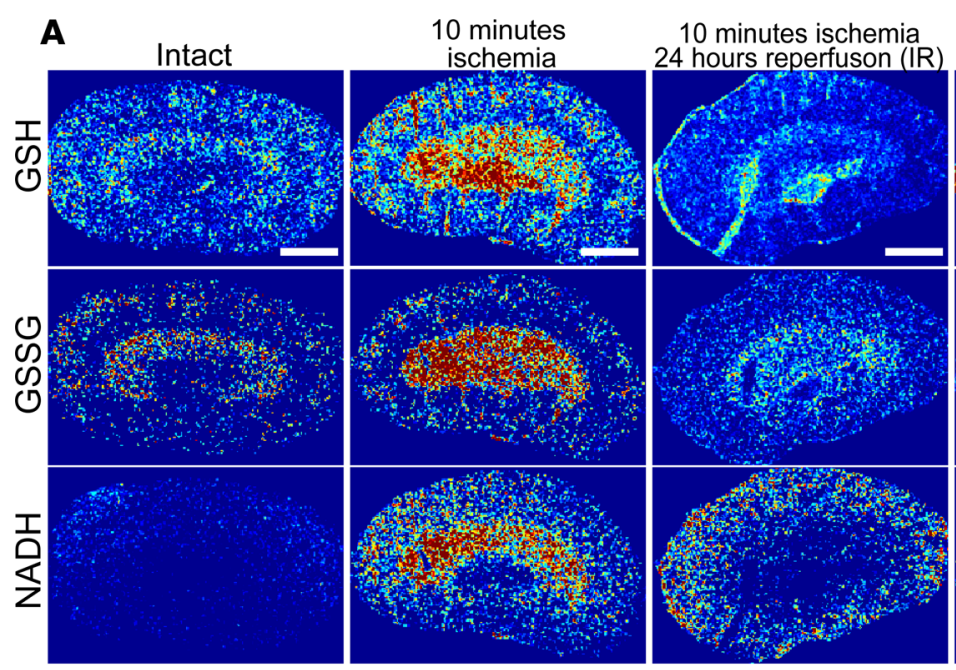

10 minutes ischemia 24 hours reperfuson + Febuxostat (IRF)

\section{B}
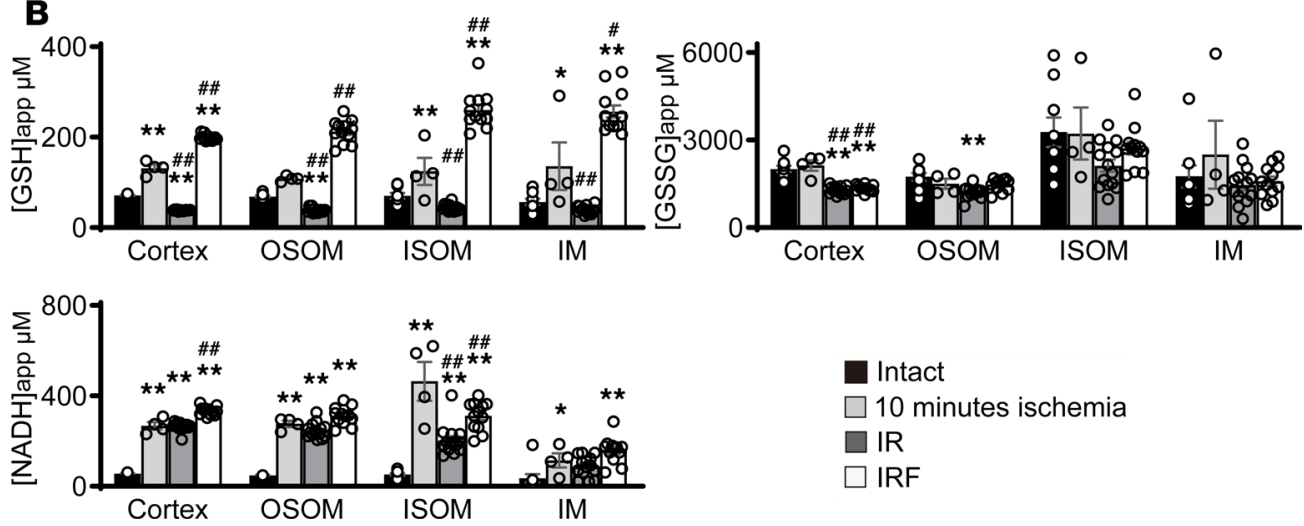

Intact

$\square 10$ minutes ischemia

$\square \mathrm{IR}$

$\square \mathrm{IRF}$

Figure 4. Redox mediators indicated ROS production in the postischemic kidney. (A) Semiquantitative mass spectrometric images of the snap-frozen murine kidney (left) subjected to 10 minutes' ischemia (middle left), IR (middle right), and IRF (right) were reconstructed at a $35-\mu \mathrm{m}$ resolution. (B) The apparent contents of metabolites (mean \pm SEM) among the data points of the renal cortex, OSOM, ISOM, and IM. ${ }^{*} P<0.05$, ${ }^{*} P<0.01$ vs. intact. $P<0.05$, \#\# $<0.01$, vs. 10 minutes' ischemia. Scale bars: $1 \mathrm{~mm} . n=5$ animals in each group for quantitative analyses. $n=9$ (intact) $n=4$ (10 minutes ischemia), $n=14$ (IR), and $n=12$ (IRF) animals for imaging MS analyses. Differences in mean values among 4 groups (the cortex, OSOM, ISOM, IM) were statistically analyzed by 1-way ANOVA and Tukey's post hoc test. GSH, reduced glutathione. GSSG, oxidized glutathione.

HPRT1 gene by using the shRNA vector was performed in HK-2 cells that were subjected to OGD and reperfusion (Figure 8, A and B). The procedure decreased the expression of HPRT1 gene by $50 \%$ in both OGD and control conditions. By silencing HPRT1, intracellular ATP decreased by $20 \%$ compared with control, and promotion of ATP resynthesis by febuxostat treatment was not observed (Figure 8C). Although the silencing of HPRT1 gene in HK-2 cells did not alter the antioxidant effect of febuxostat to suppress $\mathrm{H}_{2} \mathrm{O}_{2}$ production (Figure $8 \mathrm{D}$ ), the effect did not lead to a decrease in the expression of injury maker genes (Figure $8, \mathrm{E}$ and $\mathrm{F}$ ). These findings suggested that febuxostat exerts a protective effect against the in vitro model of IR injury by promoting the resynthesis of ATP and adenine nucleotides via an HPRT1-dependent purine salvage pathway.

\section{Discussion}

Although metabolic alterations caused by renal ischemia have been thought to play critical roles in the progression of $\operatorname{AKI}(2,3,38)$, spatiotemporal alterations of energy metabolites under ischemia leading to renal damage remain mostly unknown (39). The lack of regional information and quantitative validation of the energy and redox metabolites hampers development of an appropriate strategy to treat AKI. Transient renal ischemia in a short duration can cause $\mathrm{AKI}(4,5)$ and has a great impact on long-term prognosis of transplanted kidneys in humans (40). However, previous reports have examined renal injury that occurs 
A
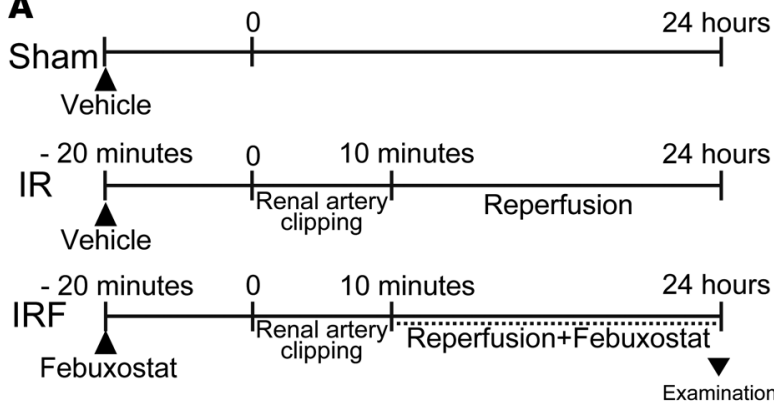

Figure 5. Febuxostat exerted protective effects against the postischemic renal injury. (A) Scheme of the present study. Renal function of mice was evaluated at 24 hours after the procedure by urine and blood test. (B) Urinary 8-OHdG/Cr, urinary NAG/Cr, and fractional excretion of sodium (FENa). ${ }^{* *} P<0.01$ sham vs. IR. ${ }^{\#} P<0.01$ IR vs. IRF. NS, not significant $(P>$ 0.05). $n=16$ animals for each group. Differences in mean values among 3 groups (sham, IR, IRF) were statistically analyzed by 1-way ANOVA and Tukey's post hoc test.
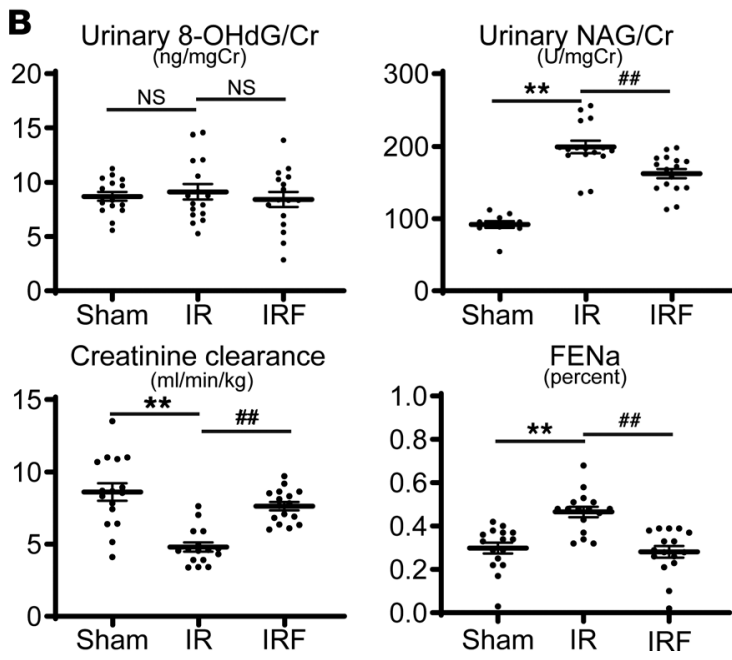

with ischemia for more than 30 minutes $(7,26)$, and the influence of renal ischemia for less than 30 minutes has not been studied enough. The present study clarified that transient ischemia for 10 minutes was sufficient to cause significant $(P<0.01)$ renal injury with increased NAG in urine and decreased $\mathrm{CCr}$, although that for 1 minute did not cause apparent renal failure. Therefore, we aimed to clarify the region-specific mechanisms of renal injury caused by short-duration ischemia by examining the spatial heterogeneity of energy and redox metabolism that accounts for postischemic renal injury. Temporal alterations of ATP and other adenine nucleotides, as well as NADH and GSH as redox regulators, were evaluated by semiquantitative MALDI-IMS combined with metabolomics.

The cortex and OSOM contain proximal and distal tubular cells, and the ISOM contains the cells constituting TALs of the loop of Henle. These cells express $\mathrm{Na}^{+} / \mathrm{K}^{+}$-ATPase for active sodium ion transportation that requires considerable amounts of ATP (41). The current technique of visualization with MALDI-IMS revealed that regional ATP concentrations were higher in the cortex and OSOM/ISOM. The finding was in good agreement with a notion that tissue ATP concentration reflects requirement of local ATP demand. ATP level in the cortex showed $45 \%$ and $84 \%$ decreases at 1 and 10 minutes after ischemia, respectively. Meanwhile, decline of ATP in the inner medulla was not so severe. The regional differences in the magnitudes of ATP drop and its recovery during and after transient ischemia were consistent with the differences in distributions of energy-metabolizing enzymes in the kidney. The proximal tubular cells in the cortex and OSOM contain relatively few glycolytic enzymes and depend on aerobic metabolism by mitochondrial respiration $(17,18)$. On the other hand, the tubular cells in the medulla, such as those in the TALs of loop of Henle and collecting ducts, contain glycolytic enzymes and have a greater capacity for anaerobic energy production during ischemia than proximal tubular cells (41). In these ways, ATP depletion during and after 10 minutes' ischemia was dominant in the cortex and OSOM, and the impact seems more severe than previously reported (42).

Importantly, ATP depletion induced by transient ischemia for 10 minutes was sustained in the cortex and OSOM even after 24 hours' reperfusion, despite the histological damage not being evident. The MALDI-IMS revealed that ATP level in the cortex decreased by $24 \%$ at the end of 24 hours' reperfusion when compared with that of sham, which should have enough impact on the function 


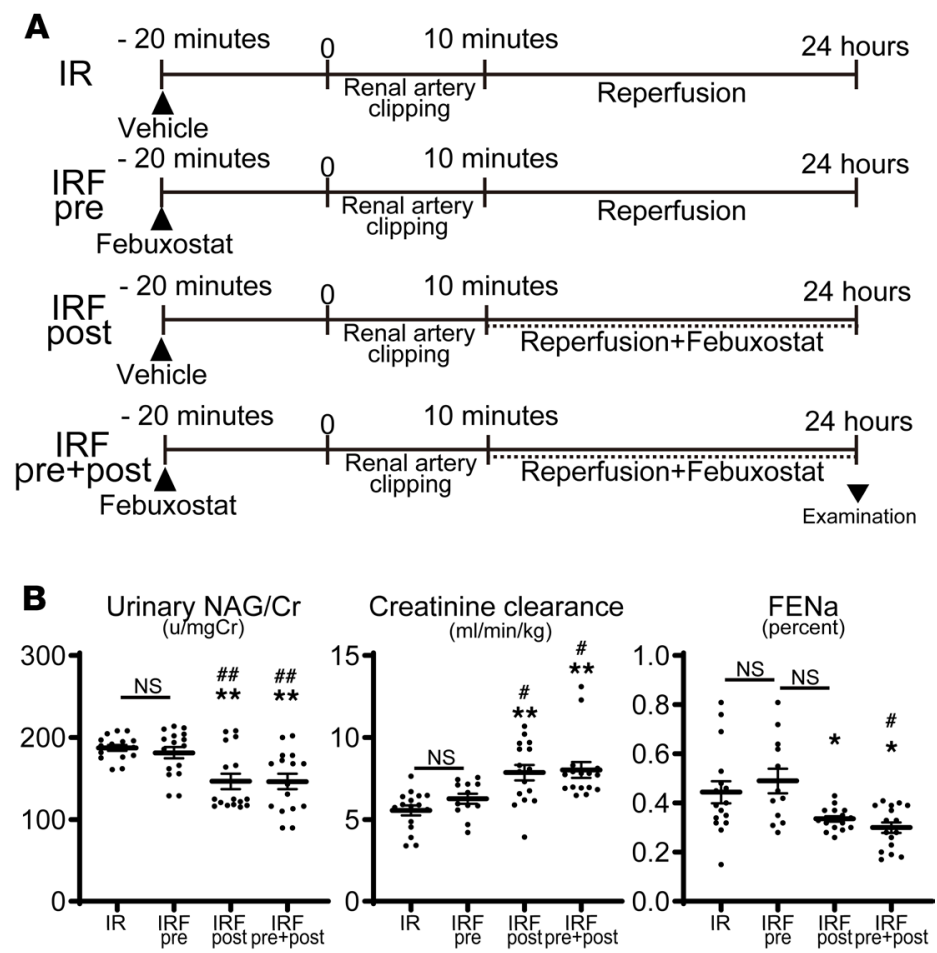

Figure 6. Renoprotective effect was more evident when febuxostat was administrated after the ischemia. (A) Scheme of the present study. Renal function of mice was evaluated at 24 hours after the procedure by urine and blood test. (B) Urinary NAG/Cr, creatinine clearance, and FENa. ${ }^{*} P<0.05$, ${ }^{*} P<0.01$ vs. IR. ${ }^{*} P<0.05$, ${ }^{\#} P<0.01$, vs. IR pre. NS, not significant $(P>0.05) . n=16$ animals for each group. Differences in mean values among 4 groups (IR, IRF pre, IRF post, IRF pre + post) were statistically analyzed by 1-way ANOVA and Tukey's post hoc test.

of proximal tubular cells (8). In AKI due to ischemia, degeneration, apoptosis, or necrosis of renal tubular cells occurs depending on the degree of ATP depletion (43). ATP depletion leads to a decrease in $\mathrm{Na}^{+} / \mathrm{K}^{+}$-ATPase activity, an increase in intracellular $\mathrm{Na}^{+}$level, and cell swelling, which may cause actin cytoskeletal alterations and detachment of tubular cells from the basement membrane $(44,45)$. Incomplete recovery of ATP can contribute to fibroblast proliferation and cause the transition to CKD, which is called AKI-CKD transition (46). Thus, sufficient ATP is required for maintenance of proximal tubular cells after transient renal ischemia $(38,47)$. From these facts, we conclude that prolonged loss of ATP after transient ischemia participates in the progression of AKI.

As for spatiotemporal alterations in thiol metabolites that contribute to attenuating oxidative stress in each region, 10 minutes' ischemia caused an increase in GSH. After reperfusion, the elevated GSH dropped in all regions of the kidney. The result suggests that the ischemia suspends constitutive ROS production and induces GSH elevation (48), and upon reperfusion, the elevated GSH ameliorates oxidative stress in all regions of the postischemic kidney. It is noteworthy that the temporal NADH alterations after reperfusion exhibited remarkable spatial heterogeneity. Sustained elevation of $\mathrm{NADH}$ after short-duration ischemia was seen in the cortex and OSOM, but not in the ISOM and inner medulla. NADH accumulation plays crucial roles in the elevation of oxidative stress after reperfusion through its potent inhibitory action on XDH (49). When accumulated $\mathrm{NADH}$ inhibits $\mathrm{XDH}, \mathrm{XOD}$ serves as an only enzyme that catalyzes the reaction of hypoxanthine to uric acid, generating massive ROS by consuming reperfused $\mathrm{O}_{2}$ (33). In addition to $\mathrm{XDH}$ inhibition, accumulated $\mathrm{NADH}$ can exert various actions as a potent reducing reagent that increases oxidative stress, such as promotion of superoxide production in mitochondria (50) and release of ferrous iron $\left(\mathrm{Fe}^{2+}\right)$ from ferritin (51). As judged by the MALDI-IMS observation that NADH elevation in the cortex and OSOM was prolonged even after reperfusion, it is not unreasonable to suggest that ROS production continues during reperfusion in these regions (52). The observation that the ISOM exhibited a reversal of $\mathrm{NADH}$ after reperfusion suggests that reperfusion-induced oxidative stress in the ISOM is not as robust as that in the cortex or OSOM, implying the vulnerability of the cortex to IR injury. Meanwhile, 8-OHdG staining showed that apparent oxidative damage 


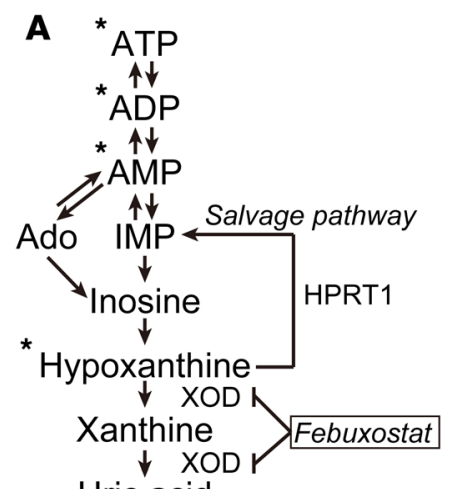

Uric acid

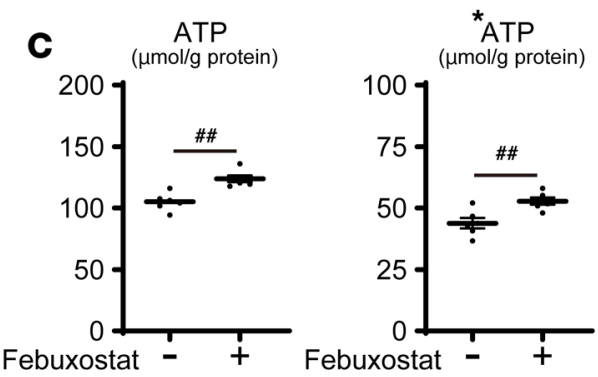

B
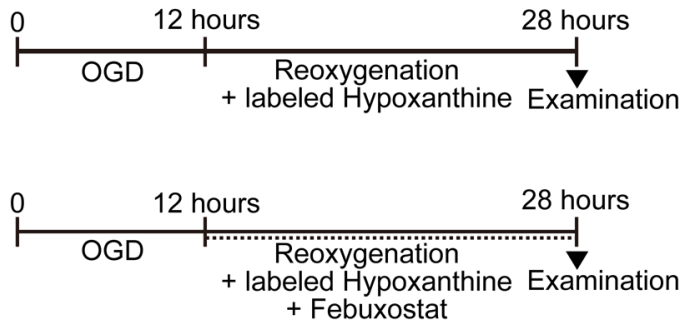

D Total adenylates

*
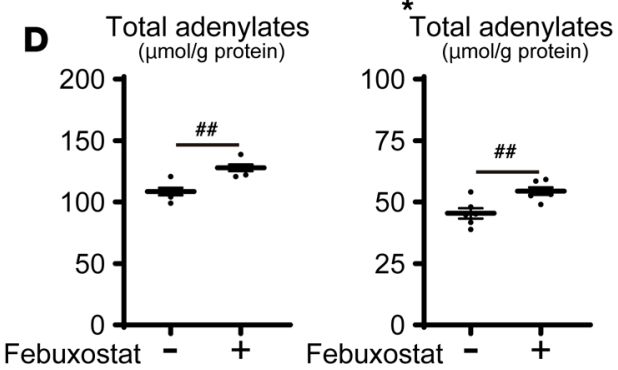

Figure 7. Febuxostat promoted ATP recovery after oxygen and glucose deprivation in cultured tubular cells. (A) Scheme of the nucleoside salvage pathway. Asterisk means stable isotope-labeled metabolites. (B) Scheme of the present study. HK-2 cells were exposed to hypoxia $\left(5 \% \mathrm{CO}_{2}, 1 \% \mathrm{O}_{2}\right)$ for 12 hours followed by 16 hours of reoxygenation $\left(5 \% \mathrm{CO}_{2}, 21 \% \mathrm{O}_{2}\right)$. During hypoxia, the culture medium was replaced with DMEM containing no glucose (OCD). During reoxygenation, the culture medium was replaced with DMEM containing glucose, $10 \%$ FBS, and $100 \mu \mathrm{M}$ labeled hypoxanthine $-{ }^{13} C_{2},{ }^{15} \mathrm{~N}$. (C) The contents of ATP and labeled ATP were measured from cell lysates. (D) The contents of total adenylates (ATP + ADP + AMP) and labeled total adenylates. ${ }^{\# \# ~} P<0.01 . n=6$ for each group. Differences in mean values were statistically analyzed by Student's 2-tailed $t$ test. HPRT1, hypoxanthine phosphoribosyl transferase 1.

after short-duration ischemia was limited, which suggests detoxification of ischemia-induced oxidative stress by the action of GSH in all regions. Collectively, these observations support a notion that AKI by short-duration ischemia includes mechanisms besides oxidative stress; we thus focused on the effects of insufficient recovery of ATP in the cortex and OSOM on the postischemic kidney.

Generally, the most common reason for insufficient recovery of ATP after ischemia is mitochondrial dysfunction that results in a decline in phosphorylation of ADP to ATP. In this case, energy charge value, which indicates the proportion of high-energy adenylates (ATP $+[0.5 \times \mathrm{ADP}])$ among total adenylates $(\mathrm{ATP}+\mathrm{ADP}+\mathrm{AMP})$, is lower (21). However, MALDI-IMS in the present study revealed that energy charge value in the cortex and OSOM after reperfusion was sufficiently recovered, although depletion of ATP was sustained in those regions. In contrast, depletion of total adenylates by 10 minutes' ischemia was not recovered in the cortex and OSOM even after 24 hours' reperfusion. These findings led us to hypothesize that depletion of total adenylates might cause incomplete recovery of ATP in the kidney after 24 hours' reperfusion. Loss of adenylates under ischemic conditions occurs by excretion of the breakdown products in the urine. After they are degraded from hypoxanthine to xanthine by XOD, they should be excreted as uric acid in the urine. However, most of the breakdown products are resynthesized to adenylates by ribosylation of hypoxanthine through the salvage pathway. There are 2 pathways to synthesize ATP: the de novo pathway and salvage pathway. Although the de novo pathway, in which IMP and adenylates are synthesized from ribose-5-phosphate, needs a great amount of ATP (47), the salvage pathway that synthesizes them from hypoxanthine needs less ATP (53). In addition, only $10 \mathrm{nmol} / \mathrm{g}$ tissue/h recovers ATP via the de novo pathway; thus, the resynthesis of adenine nucleotides via the salvage pathway is essential for yielding sufficient ATP recovery (54).

Based on these facts, we hypothesized that the resynthesis of adenylates in the postischemic kidney could be promoted by a treatment with an XOD inhibitor, febuxostat, because it can increase the tissue level of hypoxanthine (34), which serves as a substrate for the resynthesis pathway of adenine nucleotides. 
A

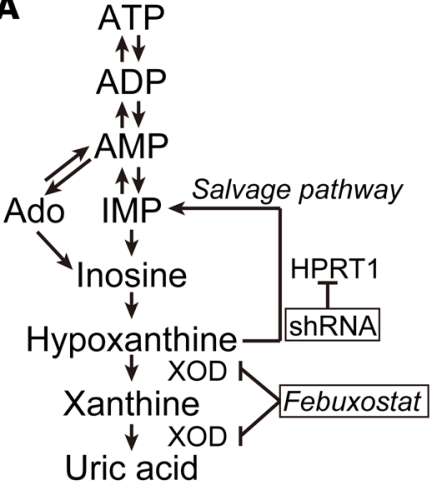

C

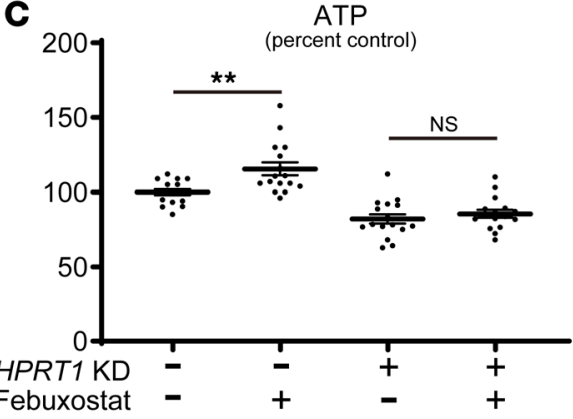

E
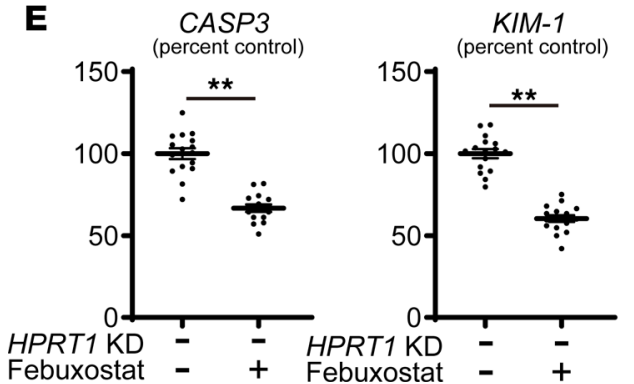

B

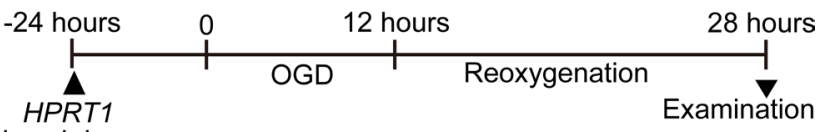

knockdown

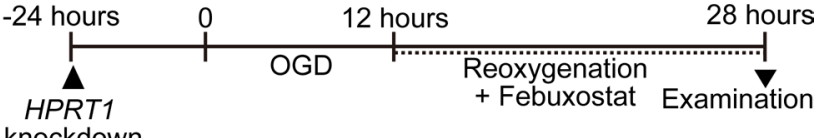

knockdown

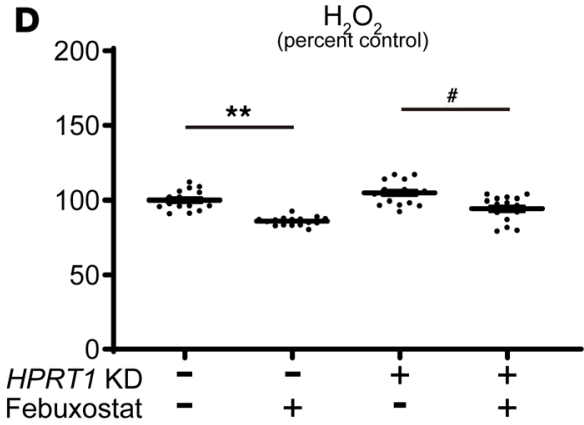

$\mathbf{F}$

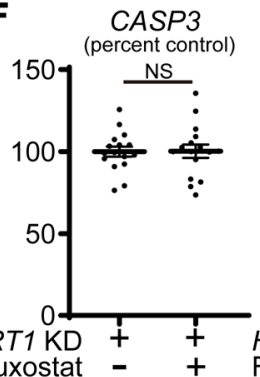

KIM-1

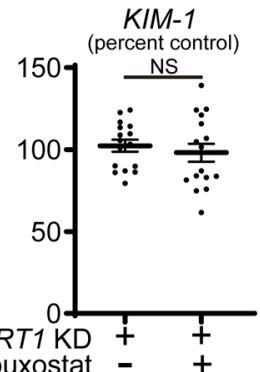

Figure 8. Febuxostat promoted resynthesis of adenine nucleotides via the HPRT1-dependent purine salvage pathway and suppressed the renal injury marker genes. (A) Scheme of the nucleoside salvage pathway. (B) Scheme of the present study. HK-2 cells were exposed to hypoxia $\left(5 \% \mathrm{CO}_{2}, 1 \% \mathrm{O}_{2}\right)$ for 12 hours followed by 16 hours of reoxygenation $\left(5 \% \mathrm{CO}_{2}, 21 \% \mathrm{O}_{2}\right)$. During hypoxia, the culture medium was replaced with DMEM containing no glucose (OGD). Transfection of shRNA against human HPRT1 and nontarget shRNA into HK-2 cells was performed 24 hours prior to OCD. (C) The contents of ATP were measured from cell lysates prepared after 16 hours of reoxygenation. Data are shown as relative values against nontarget shRNA, febuxostat-negative. (D) The contents of $\mathrm{H}_{2} \mathrm{O}_{2}$ were measured after 16 hours of reoxygenation. Data was shown as a relative value against nontarget shRNA, febuxostat-negative. (E) Gene expression of CASP3 and KIM-1 in HK-2 cells, which were exposed to OGD for 12 hours followed by 16 hours of reoxygenation and transfected with control shRNA. Data are shown as relative value against febuxostat-negative. (F) Gene expression of CASP3 and KIM-1 in HK-2 cells, which were exposed to OCD for 12 hours followed by 16 hours of reoxygenation and transfected with shRNA specific for the human HPRT1 gene. Data are shown as relative value against febuxostat-negative. ${ }^{* *} P<0.01$ febuxostat-negative vs. febuxostat-positive in control cells. ${ }^{*} P<0.05$ febuxostat-negative vs. febuxostat-positive in HPRT1-knockdown (HPRT1 KD) cells. NS, not significant $(P>0.05) . n=16$ for each group. Differences in mean values were statistically analyzed by Student's 2-tailed $t$ test.

MALDI-IMS demonstrated dramatic increases in hypoxanthine and xanthine by febuxostat treatment and showed that XOD was efficiently inhibited by febuxostat. The fact that hypoxanthine did not increase as much as xanthine supports the notion that hypoxanthine had been consumed for the resynthesis of adenine nucleotides via the salvage pathway. In addition, the experiments using HK-2 cells treated with labeled hypoxanthine, which was added during reoxygenation following OGD, confirmed that approximately $40 \%$ of intracellular ATP was generated through the salvage pathway. Previously, promoted recovery of ATP by supplementation of purine nucleobases has been shown to exert protective effects on proximal tubular cells of the porcine kidney against cellular injury by OGD (12). Thus, sustained depletion of ATP after 
transient ischemia can be ameliorated by febuxostat treatment through promoting the salvage pathway for the resynthesis of adenine nucleotides.

Suppression of superoxide production through XOD inhibition has been demonstrated as a principal organ-protective mechanism of febuxostat after renal ischemia for 30 minutes (26). Recently, febuxostat has been reported to have a renoprotective effect, independent of XOD inhibition (55). Furthermore, promotion of ATP recovery via the purine salvage pathway has been reported in the ischemic brain (56). The observation of the postischemic murine kidney with MALDI-IMS proved that febuxostat treatment significantly increased total adenylates and ATP after short-duration ischemia, which were insufficient even after 24 hours' reperfusion. In the experiments using HK-2 tubular cells, the expression of the renal injury marker genes was dramatically increased by OGD in HK-2 cells and suppressed by febuxostat treatment. Although silencing of HPRT1 in HK-2 cells did not affect the effect of febuxostat to decrease $\mathrm{H}_{2} \mathrm{O}_{2}$ production, it inhibited promotion of ATP recovery during OGD by febuxostat. As a result, the effect of febuxostat in decreasing the expression of renal injury maker genes during OGD was canceled when HPRT1 was silenced by shRNA. These findings implied that the renoprotective mechanism of febuxostat after transient ischemia was at least partially based on the promotion of ATP recovery.

In the present study, we identified crucial depletion of ATP in the cortex and OSOM, which played significant roles in postischemic renal injury after short-duration ischemia. The MALDI-IMS observation revealed spatial heterogeneity of energy and redox metabolism: the incomplete recovery of ATP and prolonged NADH elevation in the cortex and OSOM after short-duration ischemia were compatible with vulnerability of these regions to ischemia. Although sustained elevation of NADH in the cortex indicates resultant production of ROS, histological damage by oxidative stress was limited possibly because of the amelioration by consumption of GSH during reperfusion. Febuxostat promoted the resynthesis of adenine nucleotides and thus enhanced the maintenance of ATP in the postischemic kidney. Because the effect of febuxostat was canceled by silencing of HPRT1, the renoprotective effect was indicated to be exerted through the HPRT1-dependent purine salvage pathway. The novelty of the study includes the identification of prolonged ATP depletion, which played roles in renal injury after short-duration ischemia and the promotion of resynthesis of adenine nucleotides by febuxostat, which led to efficient ATP recovery and renoprotection. From these facts, mitigation of the prolonged loss of total adenylates by an XOD inhibitor would be a new therapeutic approach for postischemic AKI. Of interest is the fact that repeated episodes of AKI account for an etiology of CKD (14). Considering the AKI-CKD transition, the current results collected by MALDI-IMS not only provide deeper understanding of the pathophysiology of postischemic AKI but also shed light on the mechanism for chronic postischemic kidney disease, which deserves further investigation.

\section{Methods}

Animals and chemicals. Male C57BL/6 mice were purchased at 8 weeks of age from Charles River Laboratories (Tokyo, Japan) and maintained under specific pathogen-free conditions. Renal ischemia was produced by left renal artery occlusion using artery clips for 1 or 10 minutes. Occlusion was confirmed visually by a change in the color of the kidneys to a paler shade. Mice in the febuxostat group were injected with $0.5 \mathrm{mg} / \mathrm{kg}$ febuxostat (Teijin Pharma, Tokyo, Japan) intraperitoneally 20 minutes before the renal artery clipping (7) and were treated with drinking water containing $0.027 \mathrm{mg} / \mathrm{mL}$ of febuxostat (57). In the pretreatment group, febuxostat was injected intraperitoneally 20 minutes before the renal artery clipping, and mice were not treated with drinking water containing febuxostat. In the posttreatment group, mice were treated with drinking water containing febuxostat and not injected intraperitoneally before the renal artery clipping. The mice were sacrificed 24 hours ( $n=4$ in each group) after reperfusion and the kidneys were harvested.

MALDI-IMS. Kidneys from 8-week-old C57BL/6 mice anesthetized by isoflurane were harvested and frozen by liquid nitrogen within a second (normal sections), at a minute (ischemic sections), at 10 minutes after clamping the blood vessel, and at 40 minutes after clamping the blood vessel. Renal ischemia was induced by the application of nontraumatic microvascular clamps around the left renal artery. In the IR group, the clamps were removed after 10 minutes' ischemia. Animals were randomly assigned to the following groups: sham operated, IR with saline, and IR with febuxostat (Teijin Pharma Ltd., Tokyo, Japan). Mice were administrated $5 \mathrm{mg} / \mathrm{kg}$ intraperitoneally 20 minutes before the procedure. Mice were also administrated drinking water containing $0.027 \mathrm{mg} / \mathrm{mL}$ of febuxostat during reperfusion. Samples were collected at 24 hours. 
A MALDI-quadrupole ion trap-time of flight (TOF) mass spectrometer (iMScope: Shimadzu Corp, Kyoto, Japan) was used for data acquisition. Mass spectra acquired from each measurement point in a $35-\mu \mathrm{m}$ pitch were reconstructed as 2-dimensional heatmaps using IMAGEREVEAL MS software (Shimadzu Corp, Kyoto, Japan). After raster scanning for IMS, sections were washed with acetone and stained with hematoxylin and eosin (H\&E) according to the manufacturer's protocol (Muto Pure Chemicals, Tokyo, Japan).

Chemicals, animals, and sample collection. The chemical 9-aminoacridine (9-AA) was purchased from MilliporeSigma (Darmstadt, Germany), and 2,5-dihydroxybenzoic acid (DHB) was purchased from Bruker Daltonics (Bremen, Germany). All chemical standards were obtained from common commercial sources and were analytical or reagent grade. In the current study, mice were fed laboratory chow and allowed free access to water. The frozen kidney tissues were dissected and cryosections with a $10-\mu \mathrm{m}$ thickness prepared using a cryostat (CM 3050S; Leica Microsystems, Wetzlar, Germany). Sections were thaw-mounted on indium tin oxide- and Matsunami adhesive silane-coated slides (SI0100M, Matsunami Glass, Osaka, Japan) and dried in silica gel-containing plastic tubes. Slides were then sprayed with 9-AA (for negative ion detection; $2.5 \mathrm{mg} / \mathrm{mL}$ in $80 \%$ ethanol) or DHB (for positive ion detection; $30 \mathrm{mg} / \mathrm{mL}$ in $75 \%$ methanol, $0.2 \%$ trifluoroacetic acid) solution in preparation for MALDI-IMS.

MALDI-IMS and identification of metabolites. Shimadzu's Imaging MS Solution and IMAGEREVEAL MS software were used for MALDI-IMS data processing and statistical analyses. Mass images were acquired in positive $(100-270 \mathrm{~m} / z)$ and negative $(100-320$ and $85-300 \mathrm{~m} / z)$ ion mode, with 50 laser shots per spot. The interval for data points was $35 \mu \mathrm{m}$. Tandem MS analyses were performed to identify the structures of metabolites at the appropriate laser energy, argon gas percentage, and collision-induced dissociation energy conditions. To identify metabolites detected by MALDI-IMS, we searched the Human Metabolome Database (http://hmdb.ca) and performed tandem MS analyses, comparing tandem mass spectra with those from standard reagents. For example, we compared tandem MS fragments of $268.09 \mathrm{~m} / \mathrm{z}$ from tissue sections with those of authentic adenosine under identical analyzing conditions. An accurate mass of fragment ions $(136.06 \mathrm{~m} / \mathrm{z})$ from a kidney frozen section was identical to that of authentic adenosine.

Mass spectra acquired from each measurement point in a $35-\mu \mathrm{m}$ pitch were reconstructed as 2-dimensional heatmaps using IMAGEREVEAL MS software (Shimadzu Corp, Kyoto, Japan). After raster scanning for IMS, sections were washed with acetone and stained with H\&E according to the manufacturer's protocol (Muto Pure Chemicals, Tokyo, Japan). H\&E-stained slides were scanned with a virtual slide scanner (Nanozoomer-XR, Hamamatsu Photonics, Hamamatsu, Japan). The renal cortex, the OSOM, the ISOM, and the inner medulla were identified in each image.

Histological determination of 4 regions of the kidney. For histological analysis, we divided the kidney based on morphological findings: the renal cortex, the OSOM, the ISOM, and the inner medulla. These regions were histologically determined by observing H\&E-stained sections after raster scanning for MALDI-IMS. The cortex was identified as the outer portion of the kidney, which contains the glomerulus and the tubules. The OSOM was identified as the area where the S3 segment of proximal tubules were observable. The ISOM was identified as the area where thick limbs of the loop of Henle were located, but not proximal tubules. Sections stained with periodic acid-Schiff were also used to identify the 4 regions of the kidney.

Determination of the apparent content of metabolites based on data calibration by CE-MS. Quantitative IMS was conducted according to a previously reported method $(21,23)$ with some modifications. To compare differences in the content of metabolites in multiple tissue slices, we conducted CE-MS-based metabolome analyses of tissue lysates collected from the snap-frozen kidney samples for IMS. The CE-MS samples, weighing around $200 \mathrm{mg}$ wet weight, were grounded using a multibead shocker (model MB755U, Yasui Kikai Corp., Osaka, Japan) with a frozen disruption device and $1 \mathrm{~mL}$ methanol containing internal standards (200 $\mu \mathrm{M}$ each of L-methionine sulfone and 2-morpholinoethanesulfonic acid) was added. After addition of $0.5 \mathrm{~mL}$ of deionized water and $0.8 \mathrm{~mL}$ chloroform, the mixture was centrifuged at 20,000 $g$ for 15 minutes at $4^{\circ} \mathrm{C}$. The upper aqueous region of the sample was filtered through the $5-\mathrm{kDa}$ cutoff filter (MilliporeSigma, Tokyo, Japan) by centrifugation to remove protein components. The filtrate was lyophilized and dissolved in $200 \mu \mathrm{L}$ of deionized water containing reference compounds ( $200 \mu \mathrm{M}$ each of 3 -aminopyrrolidine and trimesate). The amounts of metabolites were normalized by kidney weight of the original tissue samples. To estimate the relative volume of each region, the kidney was divided vertically into sections with 1-mm thickness. Measured surface areas of each region in those sections were multiplied by the thickness $(1 \mathrm{~mm})$ and were summed up for quantification of the volume. The ratio of the volume of each region to the whole kidney was calculated (Supplemental Figure 2). 
Using our previous studies (23), we determined the apparent content of adenine nucleotides and the breakdown products from the signal intensity of MALDI-IMS and the amount quantified by metabolome analyses. Based on these data, apparent energy charge value of individual pixels was calculated by the following equation: apparent energy charge value $=\left(\mathrm{ATP}_{\text {app }}+\left[0.5 \times \mathrm{ADP}_{\text {app }}\right]\right) /\left(\mathrm{ATP}_{\text {app }}+\mathrm{ADP}_{\text {app }}+\mathrm{AMP}_{\text {app }}\right)$.

Analysis of biological parameters. Serum and urine samples were obtained 24 hours after reperfusion. Measurements of serum creatinine, serum sodium, urinary creatinine, urinary sodium, urinary NAG, and 8-OHdG were performed by a standard method (SRL, Tokyo, Japan). CCr was calculated by the following formula: $\mathrm{CCr}(\mathrm{mL} / \mathrm{min} / \mathrm{kg})=($ urinary creatinine $[\mathrm{mg} / \mathrm{dL}] \times$ urine volume $[\mathrm{mL} /$ day $]) /$ serum creatinine $[\mathrm{mg} / \mathrm{dL}] / 1440 /$ body weight $[\mathrm{kg}]$. NAG/Cr was calculated by the formula: NAG/Cr = urinary NAG (IU/ $\mathrm{dL}) /$ urinary creatinine $(\mathrm{mg} / \mathrm{dL})$. FENa was calculated by the formula: $\mathrm{FENa}=$ (urinary sodium $[\mathrm{mg} / \mathrm{dL}]$ $\times$ serum creatinine $[\mathrm{mg} / \mathrm{dL}]) /($ serum sodium $[\mathrm{mg} / \mathrm{dL}] \times$ urinary creatinine $[\mathrm{mg} / \mathrm{dL}]) .8-\mathrm{OHdG} / \mathrm{Cr}$ was calculated by the formula: $8-\mathrm{OHdG} / \mathrm{Cr}=$ urinary $8-\mathrm{OHdG}(\mathrm{ng} / \mathrm{dL}) /$ urinary creatinine $(\mathrm{mg} / \mathrm{dL})$.

Analysis of renal histological features. In periodic acid-Schiff staining, sections were deparaffinized, hydrated, and incubated with $1 \%$ periodic acid solution for 10 minutes. After rinsing with distilled water, sections were incubated with cold Schiff's reagent for 30 minutes. Sections were washed with sulfite solution and distilled water, then counterstained with Mayer's hematoxylin for 30 seconds and mounted. Immunohistochemical staining was performed as follows. FFPE sections with a 4- $\mu \mathrm{m}$ thickness were deparaffinized and hydrated. Antigen retrieval was performed with $10 \mathrm{mM}$ citrate buffer ( $\mathrm{pH}$ 6.0). Endogenous peroxidase was blocked with $0.3 \% \mathrm{H}_{2} \mathrm{O}_{2}$ in methanol for 30 minutes. To reduce endogenous mouse Ig staining, sections were incubated with mouse IgG blocking reagent (Biocare Medical, Pacheco, California, USA) for 1 hour. After washes with PBS, sections were incubated with anti-8-OHdG monoclonal antibody (N45.1, Japan Institute for the Control of Aging, Fukuroi, Japan), diluted $10 \mu \mathrm{g} / \mathrm{mL}$ for 1 hour.

Quantitative analysis of renal histological features. Histological features were evaluated 24 hours after reperfusion. To evaluate the histological features in the ischemic kidneys, all images were scanned by the Nanozoomer-XR (Hamamatsu Photonics K.K., Shizuoka, Japan). Tubulointerstitial injury was evaluated using 10 randomly selected fields of the cortex per cross section in each of the 4 kidneys. The score was graded ( 0 to 5) on the basis of the percentage of tubular cellularity, basement membrane thickening, cell infiltration, dilation, atrophy, sloughing, or interstitial widening as follows: 0 , no change; $1,10 \%$ tubulointerstitial injury; 2 , $10 \%-25 \%$ injury; $3,25 \%-50 \%$ injury; $4,50 \%-75 \%$ injury; and $5,75 \%-100 \%$ injury. The positive expression of 8-OHdG was analyzed by using Adobe Photoshop CC 2015. For each section, 10 nuclei from 3 fields of the cortex were randomly selected from 4 kidneys. To evaluate the positive expression of 8-OHdG immunostaining, nucleus density in positive cells was evaluated by using Photoshop CC 2015.

Cell culture experiments using HK-2 tubular cells. HK-2, which is an immortalized proximal tubule epithelial cell line, was obtained from the American Type Culture Collection (Manassas, Virginia, USA). For the OGD group, the culture medium was replaced with DMEM containing no glucose prior to the induction of hypoxia. Cells were cultured in a hypoxia incubator and exposed to hypoxia $\left(5 \% \mathrm{CO}_{2}, 1 \% \mathrm{O}_{2}\right.$, and $94 \%$ $\mathrm{N}_{2}$ ) for 12 hours followed by 24 hours of reoxygenation $\left(5 \% \mathrm{CO}_{2}, 21 \% \mathrm{O}_{2}\right.$, and $\left.74 \% \mathrm{~N}_{2}\right)$. During reoxygenation, the culture medium was replaced with DMEM containing glucose and FBS. For the febuxostat group, HK-2 cells were treated with $100 \mu \mathrm{M}$ febuxostat after OGD. Silencing of HPRT1 was induced by transfection of shRNA against human HPRT1 and nontarget shRNA into HK-2 cells using Lipofectamine 3000 (Thermo Fisher Scientific, Waltham, Massachusetts, USA ).

Determination of stable isotope-labeled metabolites in cultured HK-2 cells by CE-TOF-MS. The culture medium was replaced with DMEM containing hypoxanthine- ${ }^{13} \mathrm{C}_{2},{ }^{15} \mathrm{~N}$ during reoxygenation. The cells were washed in 5\% mannitol (Wako Pure Chemicals, Osaka, Japan) and then plunged into methanol containing internal standards (200 $\mu \mathrm{M}$ each of methionine sulfone for cations, 2-morholinoethanesulfonic acid for anions). Metabolites were extracted from cell lysates with a solvent composed of methanol, distilled water, and chloroform (methanol/water/chloroform $=1: 0.5: 1[v / v / v]$ ). After centrifugation at 20,000 $g$ at $4^{\circ} \mathrm{C}$ for 15 minutes, the water and methanol layer were filtered through a 5-kDa cutoff filter (Human Metabolome Technologies, Tsuruoka, Japan). Then, the filtrate was lyophilized and dissolved in deionized water containing reference compounds $(200 \mu \mathrm{M}$ each of 3-aminopyrrolidine and trimesate) to serve as samples analyzed by CE-TOF-MS according to our previous methods $(25,58,59)$.

Estimation of ATP, $\mathrm{H}_{2} \mathrm{O}_{2}$ production, and gene expression. $\mathrm{H}_{2} \mathrm{O}_{2}$ production was assessed using Amplex Red Hydrogen Peroxide/Peroxidase Assay Kit (Thermo Fisher Scientific, Waltham, Massachusetts, USA) according to the manufacturer's protocol. ATP production was estimated using ATP Bioluminescence 
Assay Kit CLS II (Roche Applied Science, Penzberg, Germany) according to the manufacturer's protocol. The cell number was estimated using Hoechst 33342 (Dojindo, Kumamoto, Japan), and its fluorescence signal (excitement: $485 \mathrm{~nm}$; emission: $630 \mathrm{~nm}$ ) was read using a microplate reader (Synergy4, BioTek Instruments, Winooski, Vermont, USA). ATP and $\mathrm{H}_{2} \mathrm{O}_{2}$ values were normalized by the cell number, which was estimated by Hoechst 33342 staining.

Total RNA was extracted using a commercially available kit (74104, RNeasy Mini Kit; QIAGEN, Venlo, The Netherlands), and cDNA was prepared by reverse transcription (RR037A, Primescript RT reagent kit, TaKaRa Bio, Otsu, Japan). Subsequently, real-time PCR was performed using an Applied Biosystems 7500 Fast Real-Time PCR system and SYBR Premix Ex Taq II, according to the manufacturer's instructions. The mRNA levels were examined, and the results were corrected by those of RPS18 and are shown as a relative expression ratio against control. All primers were prepared by TaKaRa Bio. Primer sequences are presented in Supplemental Table 9.

Statistics. Differences in mean values among more than 3 groups were statistically analyzed by 1 -way ANOVA and Tukey's post hoc test. Differences between 2 groups were analyzed by Student's 2-tailed $t$ test. $P<0.05$ was considered statistically significant.

Study approval. This study was approved by the Ethics Committee of the laboratory animal center, Keio University School of Medicine (approval number 14004). All animal experiments were approved by the local ethics committee and were conducted in accordance with domestic law on the protection of laboratory animals, which is based on the NIH Guide for the Care and Use of Laboratory Animals (National Academies Press, 2011).

\section{Author contributions}

$\mathrm{KF}, \mathrm{AK}, \mathrm{KM}$, and M Suematsu designed the research. KF, AK, KM, M Sato, TH, and NH performed the experiments; $\mathrm{KF}, \mathrm{AK}$, and $\mathrm{KM}$ analyzed data; and YK established infrastructure of metabolomics to determine stable isotope-labeled metabolites with funding support. $\mathrm{KF}, \mathrm{AK}, \mathrm{KM}$, and M Suematsu wrote the paper. KF, AK, KM, M Sato, AH, H Inoue, MR, MT, TH, NH, YK, H Itoh, and M Suematsu read the manuscript critically; $\mathrm{H}$ Itoh and $\mathrm{M}$ Suematsu supervised the research.

\section{Acknowledgments}

This work was supported by the Japan Society for the Promotion of Science, Grant-in-Aid for Scientific Research with grant numbers JP26460920 to KM, JP16K07203 to AK, and JP16K15471 to H Itoh. Imaging MS infrastructure was established by JST ERATO Suematsu Gas Biology (FY2010-FY2015, to M Suematsu). Metabolomics infrastructure to determine metabolites labeled with stable isotopes in cultured cells was supported by AMED-CREST (JP17gm0710010 to YK).

Address correspondence to: Kazutoshi Miyashita (corresponding author), Department of Internal Medicine, School of Medicine, Keio University, 35 Shinanomachi, Shinjuku-ku, Tokyo, Japan, 160-8582. Phone: 81.3.5363.3878; Email: miyakaz@z6.keio.jp; or Akiko Kubo (co-corresponding author), Department of Biochemistry, School of Medicine, Keio University, 35 Shinanomachi, Shinjuku-ku, Tokyo, Japan, 160-8582. Phone: 81.3.5363.3461; Email: yuba@a3.keio.jp.

1. Mehta RL, et al. Acute Kidney Injury Network: report of an initiative to improve outcomes in acute kidney injury. Crit Care. 2007;11(2):R31

2. Murugan R, Kellum JA. Acute kidney injury: what's the prognosis? Nat Rev Nephrol. 2011;7(4):209-217.

3. Bonventre JV, Yang L. Cellular pathophysiology of ischemic acute kidney injury. J Clin Invest. 2011;121(11):4210-4221.

4. Tujjar O, et al. Acute kidney injury after cardiac arrest. Crit Care. 2015;19:169.

5. O'Neal JB, Shaw AD, Billings FT. Acute kidney injury following cardiac surgery: current understanding and future directions. Crit Care. 2016;20(1):187.

6. Johnson KJ, Weinberg JM. Postischemic renal injury due to oxygen radicals. Curr Opin Nephrol Hypertens. 1993;2(4):625-635.

7. Doi K, Suzuki Y, Nakao A, Fujita T, Noiri E. Radical scavenger edaravone developed for clinical use ameliorates ischemia/ reperfusion injury in rat kidney. Kidney Int. 2004;65(5):1714-1723.

8. Lieberthal W, Menza SA, Levine JS. Graded ATP depletion can cause necrosis or apoptosis of cultured mouse proximal tubular cells. Am J Physiol. 1998;274(2):F315-F327.

9. Szeto HH, et al. Mitochondria-targeted peptide accelerates ATP recovery and reduces ischemic kidney injury. J Am Soc Nephrol. 2011;22(6):1041-1052.

10. Siegel NJ, et al. Enhanced recovery from acute renal failure by the postischemic infusin of adenine nucleotides and magnesium 
chloride in rats. Kidney Int. 1980;17(3):338-349.

11. Rajakumar SV, et al. Deficiency or inhibition of CD73 protects in mild kidney ischemia/reperfusion injury. Transplantation. 2010;90(12):1260-1264.

12. Szoleczky P, et al. Identification of agents that reduce renal hypoxia-reoxygenation injury using cell-based screening: purine nucleosides are alternative energy sources in LLC-PK1 cells during hypoxia. Arch Biochem Biophys. 2012;517(1):53-70.

13. de Seigneux S, Martin PY. Preventing the progression of AKI to CKD: the role of mitochondria. J Am Soc Nephrol. 2017;28(5):1327-1329.

14. Venkatachalam MA, Weinberg JM, Kriz W, Bidani AK. Failed tubule recovery, AKI-CKD transition, and kidney disease progression. J Am Soc Nephrol. 2015;26(8):1765-1776.

15. Zager RA, Gmur DJ, BredL CR, Eng MJ, Fisher L. Regional responses within the kidney to ischemia: assessment of adenine nucleotide and catabolite profiles. Biochim Biophys Acta. 1990;1035(1):29-36.

16. Siragy HM, Linden J. Sodium intake markedly alters renal interstitial fluid adenosine. Hypertension. 1996;27(3 Pt 1):404-407.

17. Uchida S, Endou H. Substrate specificity to maintain cellular ATP along the mouse nephron. Am J Physiol. 1988;255(5 Pt 2):F977-F983.

18. Guder WG, Ross BD. Enzyme distribution along the nephron. Kidney Int. 1984;26(2):101-111.

19. Kubo A, Kajimura M, Suematsu M. Matrix-Assisted laser desorption/ionization (MALDI) imaging mass spectrometry (IMS): a challenge for reliable quantitative analyses. Mass Spectrom (Tokyo). 2012;1(1):A0004.

20. Morikawa T, et al. Hypoxic regulation of the cerebral microcirculation is mediated by a carbon monoxide-sensitive hydrogen sulfide pathway. Proc Natl Acad Sci USA. 2012;109(4):1293-1298.

21. Hattori K, et al. Paradoxical ATP elevation in ischemic penumbra revealed by quantitative imaging mass spectrometry. Antioxid Redox Signal. 2010;13(8):1157-1167.

22. Kubo A, et al. Semi-quantitative analyses of metabolic systems of human colon cancer metastatic xenografts in livers of superimmunodeficient NOG mice. Anal Bioanal Chem. 2011;400(7):1895-1904.

23. Yamamoto $\mathrm{T}$, et al. Reduced methylation of PFKFB3 in cancer cells shunts glucose towards the pentose phosphate pathway. Nat Commun. 2014;5:3480.

24. Yamazoe S, et al. Large-area surface-enhanced Raman spectroscopy imaging of brain ischemia by gold nanoparticles grown on random nanoarrays of transparent boehmite. ACS Nano. 2014;8(6):5622-5632.

25. Shiota M, et al. Gold-nanofève surface-enhanced Raman spectroscopy visualizes hypotaurine as a robust anti-oxidant consumed in cancer survival. Nat Commun. 2018;9(1):1561.

26. Tsuda H, et al. Febuxostat suppressed renal ischemia/reperfusion injury via reduced oxidative stress. Biochem Biophys Res Commun. 2012;427(2):266-272.

27. Haga Y, et al. Disruption of xanthine oxidoreductase gene attenuates renal ischemia reperfusion injury in mice. Life Sci. 2017;182:73-79

28. Bian A, et al. Downregulation of autophagy is associated with severe ischemia/reperfusion-induced acute kidney injury in overexpressing C-reactive protein mice. PLoS One. 2017;12(9):e0181848.

29. Gallazzini M, Burg MB. What's new about osmotic regulation of glycerophosphocholine. Physiology (Bethesda). 2009;24:245-249.

30. Osswald H, Schmitz HJ, Kemper R. Tissue content of adenosine, inosine and hypoxanthine in the rat kidney after ischemia and postischemic recirculation. Pflugers Arch. 1977;371(1-2):45-49.

31. Shanley PF, Brezis M, Spokes K, Silva P, Epstein FH, Rosen S. Hypoxic injury in the proximal tubule of the isolated perfused rat kidney. Kidney Int. 1986;29(5):1021-1032.

32. Shanley PF, Brezis M, Spokes K, Silva P, Epstein FH, Rosen S. Transport-dependent cell injury in the S3 segment of the proximal tubule. Kidney Int. 1986;29(5):1033-1037.

33. Suzuki H, et al. Prostaglandin E1 abrogates early reductive stress and zone-specific paradoxical oxidative injury in hypoperfused rat liver. J Clin Invest. 1994;93(1):155-164.

34. Lasley RD, Ely SW, Berne RM, Mentzer RM. Allopurinol enhanced adenine nucleotide repletion after myocardial ischemia in the isolated rat heart. J Clin Invest. 1988;81(1):16-20.

35. Osada Y, et al. Hypouricemic effect of the novel xanthine oxidase inhibitor, TEI-6720, in rodents. Eur J Pharmacol. 1993;241(23):183-188.

36. Linas SL, Whittenburg D, Repine JE. Role of xanthine oxidase in ischemia/reperfusion injury. Am J Physiol. 1990;258(3 Pt 2):F711-F716.

37. Ahmadinejad F, Geir Møller S, Hashemzadeh-Chaleshtori M, Bidkhori G, Jami MS. Molecular mechanisms behind free radical scavengers function against oxidative stress. Antioxidants (Basel). 2017;6(3):E51

38. Szeto HH. Pharmacologic approaches to improve mitochondrial function in AKI and CKD. J Am Soc Nephrol. 2017;28(10):2856-2865.

39. Siegel NJ, Avison MJ, Reilly HF, Alger JR, Shulman RG. Enhanced recovery of renal ATP with postischemic infusion of ATP-MgCl2 determined by 31P-NMR. Am J Physiol. 1983;245(4):F530-F534.

40. Tennankore KK, Kim SJ, Alwayn IP, Kiberd BA. Prolonged warm ischemia time is associated with graft failure and mortality after kidney transplantation. Kidney Int. 2016;89(3):648-658.

41. Katz AI, Doucet A, Morel F. Na-K-ATPase activity along the rabbit, rat, and mouse nephron. Am J Physiol. 1979;237(2):F114-F120.

42. Arnold PE, Van Putten VJ, Lumlertgul D, Burke TJ, Schrier RW. Adenine nucleotide metabolism and mitochondrial Ca2+ transport following renal ischemia. Am J Physiol. 1986;250(2 Pt 2):F357-F363.

43. Devarajan P. Update on mechanisms of ischemic acute kidney injury. J Am Soc Nephrol. 2006;17(6):1503-1520.

44. Molitoris BA, Geerdes A, McIntosh JR. Dissociation and redistribution of $\mathrm{Na}+, \mathrm{K}(+)$-ATPase from its surface membrane actin cytoskeletal complex during cellular ATP depletion. J Clin Invest. 1991;88(2):462-469.

45. Molitoris BA, Dahl R, Hosford M. Cellular ATP depletion induces disruption of the spectrin cytoskeletal network. Am J Physiol. 1996;271(4 Pt 2):F790-F798. 
46. Mount PF, Power DA. Balancing the energy equation for healthy kidneys. J Pathol. 2015;237(4):407-410.

47. Zimmer HG, Trendelenburg C, Kammermeier H, Gerlach E. De novo synthesis of myocardial adenine nucleotides in the rat. Acceleration during recovery from oxygen deficiency. Circ Res. 1973;32(5):635-642.

48. Simonson SG, Zhang J, Canada AT, Su YF, Benveniste H, Piantadosi CA. Hydrogen peroxide production by monoamine oxidase during ischemia/reperfusion in the rat brain. J Cereb Blood Flow Metab. 1993;13(1):125-134.

49. Sanders SA, Eisenthal R, Harrison R. NADH oxidase activity of human xanthine oxidoreductase--generation of superoxide anion. Eur J Biochem. 1997;245(3):541-548.

50. Kussmaul L, Hirst J. The mechanism of superoxide production by NADH:ubiquinone oxidoreductase (complex I) from bovine heart mitochondria. Proc Natl Acad Sci USA. 2006;103(20):7607-7612.

51. Topham R, Goger M, Pearce K, Schultz P. The mobilization of ferritin iron by liver cytosol. A comparison of xanthine and NADH as reducing substrates. Biochem J. 1989;261(1):137-143.

52. Suematsu M, et al. Early midzonal oxidative stress preceding cell death in hypoperfused rat liver. Gastroenterology. 1992;103(3):994-1001.

53. Murray AW. The biological significance of purine salvage. Annu Rev Biochem. 1971;40:811-826.

54. Dow JW, Nigdikar S, Bowditch J. Adenine nucleotide synthesis de novo in mature rat cardiac myocytes. Biochim Biophys Acta. $1985 ; 847(2): 223-227$.

55. Kojima S, et al. Febuxostat for Cerebral and CaRdiorenovascular Events PrEvEntion StuDy. Eur Heart J. 2019;40(22):1778-1786.

56. Faller KME, Leach J, Johnston P, Holmes WM, Macrae IM, Frenguelli BG. Proof of concept and feasibility studies examining the influence of combination ribose, adenine and allopurinol treatment on stroke outcome in the rat. Brain and Neuroscience Advances. 2017;1:1-13

57. Nomura J, et al. Xanthine oxidase inhibition by febuxostat attenuates experimental atherosclerosis in mice. Sci Rep. 2014;4:4554.

58. Ohmura M, et al. Impacts of CD44 knockdown in cancer cells on tumor and host metabolic systems revealed by quantitative imaging mass spectrometry. Nitric Oxide. 2015;46:102-113.

59. Tohyama S, et al. Glutamine oxidation is indispensable for survival of human pluripotent stem cells. Cell Metab. 2016;23(4):663-674 